\title{
GESTION RESPONSABLE DES RESSOURCES HUMAINES : ÉVALUATION THÉORIQUE ET ANALYSE DU DISCOURS DES BANQUES CANADIENNES SUR LEUR PRATIQUE Lovasoa Ramboarisata, Andrée de Serres et Corinne Gendron
}

\section{ESKA | « Revue internationale de psychosociologie »}

2008/33 Vol. XIV | pages 225 à 258

ISSN 1260-1705

ISBN 2747214667

\section{Article disponible en ligne à l'adresse :}

https://www.cairn.info/revue-internationale-depsychosociologie-2008-33-page-225.htm

Distribution électronique Cairn.info pour ESKA.

(C) ESKA. Tous droits réservés pour tous pays.

La reproduction ou représentation de cet article, notamment par photocopie, n'est autorisée que dans les limites des conditions générales d'utilisation du site ou, le cas échéant, des conditions générales de la licence souscrite par votre établissement. Toute autre reproduction ou représentation, en tout ou partie, sous quelque forme et de quelque manière que ce soit, est interdite sauf accord préalable et écrit de l'éditeur, en dehors des cas prévus par la législation en vigueur en France. Il est précisé que son stockage dans une base de données est également interdit. 


\title{
GESTION RESPONSABLE DES RESSOURCES HUMAINES : EVALUATION THEORIQUE ET ANALYSE DU DISCOURS DES BANQUES CANADIENNES SUR LEUR PRATIQUE
}

\author{
Lovasoa Ramboarisata ${ }^{277}$ \\ Andrée De Serres ${ }^{278}$, \\ Corinne Gendron ${ }^{279}$
}

Comme représentation du rapport entre l'entreprise et la société, la responsabilité sociale d'entreprise (RSE), bien qu'elle ait été définie de différentes manières ${ }^{280}$, suscite l'intérêt de différents acteurs, comme en témoignent les initiatives politiques $^{281}$, sociales ${ }^{282}$ et économiques ${ }^{283}$ qui la favorisent ou qui la scrutent.

${ }^{277}$ MBA, École des sciences de la gestion, Université du Québec à Montréal. Lovasoa Ramboarisata est
candidate au doctorat en administration à l'École des sciences de la gestion de l'Université du Québec à
Montréal et étudiante chercheure au Groupe interdisciplinaire de recherche en éthique financière
(GIREF) et à la Chaire de responsabilité sociale et de développement durable. ramboarisata.lovasoa@courrier.uqam.ca

${ }^{278}$ LL.L, MBA, Ph.D, École des sciences de la gestion, Université du Québec à Montréal , Andrée De Serres est professeure au Département stratégie des affaires de l'Université du Québec à Montréal, directrice du programme de MBA pour cadres en financement d'entreprise, Co-directrice du Groupe interdisciplinaire de recherche en éthique financière (GIREF) et chercheure associée à la Chaire de responsabilité sociale et de développement durable. deserres.andree@uqam.ca

${ }^{279}$ LL.B, MBA, Ph.D, École des sciences de la gestion, Université du Québec à Montréal, Corinne Gendron est professeure au Département de stratégie et responsabilité sociale et environnementale de l'Université du Québec à Montréal et titulaire de la Chaire de responsabilité sociale et de développement durable. gendron.corinne@uqam.ca

${ }^{280}$ Notons qu'il existe différentes représentations de la RSE (Champion et al., 2005). Dans le monde des affaires, on la considère généralement comme un modèle de gestion. Comme l'explique SahlinAndersson (2006), comme modèle de gestion, la RSE se traduit par l'adoption, par les organisations, de pratiques prenant en considération les attentes sociales et qui paraissent légitimes, modernes et attrayantes aux yeux de leurs employés, collaborateurs, clients et autres partenaires. Une telle représentation correspond davantage à la vision anglo-saxonne de la RSE et demeure encore proche de la définition de Bowen (1953), considéré comme le père de la RSE et qui l'a définie, il y a plus d'une cinquantaine d'années comme étant les obligations des dirigeants d'entreprises de formuler des stratégies, de prendre des décisions et de suivre des plans d'actions favorables aux objectifs et aux valeurs de la société.

${ }^{281}$ Les législateurs et les gouvernements de différents pays se sont mobilisés de diverses façons (par exemple, consultation publique au Québec; adoption de lois en France, au Canada et aux Etats-Unis; création de partenariats avec les entreprises au Royaume-Uni et en Hollande, etc.) pour favoriser les pratiques liées à la RSE (Gendron et al., 2004; De Serres, 2005).

${ }^{282}$ Les activités de groupes, d'organismes non gouvernementaux et d'acteurs de la société civile, qui se sont données comme mission d'exercer une surveillance, un contrôle serré et une influence pressante sur les actions des entreprises privées se sont beaucoup multipliées. Par exemple, des organismes comme Banktrack, Rainforest Action Network, Friends of the Earth scrutent continuellement les 
Comme champ de connaissance, la RSE a bénéficié depuis les trois dernières décennies d'attention continue se traduisant par bon nombre d'initiatives académiques ${ }^{284}$. Mais malgré l'intérêt persistant qu'on y accorde, le seul consensus qui semble exister concernant la RSE est son caractère flou. La gestion responsable des ressources humaines ou la responsabilité sociale envers les employés, considérée comme une dimension incontournable de la RSE, s'expose aussi à cette même problématique. Des efforts académiques ${ }^{285}$ ont été investis au cours des dernières années pour «repenser la gestion des ressources humaines (GSRH) » afin de tenir compte de l'impératif éthique et de RSE. D'autre part, l'imposante théorisation de la performance sociale d'entreprise, fondée sur l'éthique et la théorie normative des parties prenantes ${ }^{286}$, place les travailleurs parmi les principaux ayants droit aux intérêts aussi légitimes que les rentiers de la firme. Du côté pratique, les critères de performance en matière de RSE s'imposant tant par les obligations légales ${ }^{287}$ que par les normes volontaires ${ }^{288}$ incluent ceux liés aux thématiques de l'emploi et du respect des droits des travailleurs. Le discours des entreprises sur leurs pratiques responsables (voir par exemple De Serres et al, 2006; Vuontisjarvi, 2006) indique aussi que se préoccuper des attentes de leurs salariés et des enjeux les concernant (par exemple, la santé et la sécurité, le développement professionnel, la diversité de la main d'œuvre, etc.) est la manifestation d'un comportement responsable, au même titre que l'intégration des

activités des institutions bancaires dans le monde.

${ }^{283}$ Outre les pratiques des entreprises au niveau local, on a vu se multiplier des initiatives impliquant des acteurs économiques au niveau international (par exemple, le rassemblement autour d'expérimentations telles le World Business Council for Sustainable Development, le Global Compact, la Global Reporting Initiative, la ISO 26000, etc.).

${ }^{284}$ Ces initiatives incluent la création de division consacrée à la RSE dans les associations académiques (par exemple, la division RSE de l'Association des sciences administratives du Canada, etc.), les numéros spéciaux des journaux ou revues académiques sur ce thème (par exemple, la Revue Gestion, le Journal of Management Studies qui ont consacré un numéro spécial sur ce thème en 2006), le financement accru de programmes de recherche traitant de la RSE, la création de regroupements ou de réseaux de chercheurs travaillant dans le domaine de la RSE, la tenue de rencontres académiques récurrentes sur la RSE, l'inclusion de cours sur la RSE dans les différents programmes d'études, etc.

${ }^{285}$ Par exemple, en 2005, l'Association franchophone de gestion des ressources humaines (AGSRH) s'est fixée le suivant comme thème de son congrès annuel : " (Ré)concilier l'économique et le social ».

${ }^{286}$ Cette théorisation a été initialement introduite vers la fin des années 1970 dans quelques travaux fondateurs (Sethi, 1975; Preston et Post, 1974) et développée notamment par les travaux de Carroll (1979) d'Aupperle et al. (1985) et de Wartick et Cochran (1985), ensuite synthétisée sous forme d'un cadre théorique intégrateur par Wood (1991) et formulée en termes opératoires par Clarkson (1995).

${ }^{287}$ Par exemple, en France, le décret d'application de l'article 116 de la Loi sur les Nouvelles Régulations Économiques de 2001 spécifie les renseignements à caractère social que les entreprises qui y sont soumises doivent annexer à leur rapport de gestion. Ces renseignements incluent ceux touchant la main d'œuvre et renforcent les exigences de la Loi sur le bilan social de 1977 relatives aux ressources humaines.

${ }^{288}$ Par exemple, les indices sociaux tels les séries FTSE4GOOD, les regroupements d'entreprises tels la Canadian Business for Social Responsibility et les référentiels tels la Global Reporting Initiative, identifient des indicateurs de performance liés à la responsabilité envers les travailleurs comme faisant partie des critères que devraient respecter les entreprises qui s'y inscrivent. 
considérations environnementales, communautaires et éthiques. Pourtant, à l'instar de la RSE dont elle constitue une dimension importante, la gestion responsable des ressources humaines ou la responsabilité sociale envers les employés reste à éclaircir.

La question qui nous intéresse plus spécifiquement est la suivante : la RSE, en théorie et en pratique, permet-elle de repenser la gestion stratégique des ressources humaines (GSRH). Notre objectif est donc double. Il s'agit d'une part d'analyser dans quelles mesures l'évolution des connaissances sur la RSE (la théorie) offre une voie de renouvellement de la conceptualisation de la GSRH et d'autre part, d'examiner si les initiatives favorisées par les entreprises (la pratique) pour opérationnaliser ce qu'elles conçoivent comme leur responsabilité envers les employés peuvent être source de transformations transcendant la finalité strictement utilitaire. Afin de répondre à ce double objectif, nous procéderons d'abord à une discussion théorique (Partie 1), nourrie d'une revue de la littérature sur les thèmes de la RSE et de la GSRH, et évaluerons le potentiel d'utilisation des connaissances sur la RSE au renouvellement de celles sur la GSRH. Par la suite, nous nous baserons sur les résultats d'une étude empirique ayant porté sur les banques canadiennes (Partie 2) ${ }^{289}$ pour évaluer dans quelles mesures les pratiques actuelles de RSE qui prédominent dans les organisations pourraient induire des changements quant aux relations de celles-ci avec leurs employés.

\section{RENOUVELER LA GSRH A TRAVERS LA RSE}

L'objectif de cette première section est d'abord de fournir un bref portrait respectif de l'évolution de la littérature sur la RSE et de celle sur la GSRH. Il s'agit, par la suite, d'évaluer si et en quoi les connaissances sur la RSE permettent de renouveler celles sur la GSRH.

Comme nous le présenterons dans la revue de littérature ci-après, jusqu'à la fin des années 1990, l'analyse de la RSE était principalement fondée sur des approches managériales traditionnelles (par exemple, les théories contractuelles de la firme, la théorie de dépendance des ressources, l'éthique des affaires, l'approche des parties prenantes) et adoptait une posture fortement prescriptive, à visées instrumentale et normative. Depuis le début des années 2000, les contributions des chercheurs francophones et scandinaves, ont fortement questionné les approches traditionnelles et favorisé les théories institutionnelles économiques et les approches sociologiques afin de se distancer des visées instrumentale et normative et pour proposer un nouvel éclairage davantage empirique. Concernant les connaissances sur la GSRH, comme notre revue l'indiquera, la littérature demeure

${ }^{289}$ Fruit d'une recherche commanditée par le Programme des services financiers et politique publique de Schulich Business School (Université York). 
dominée par les approches stratégiques traditionnelles, notamment la théorie de dépendance des ressources. Malgré l'existence d'une part d'une tournure éthique, fondée sur l'éthique philosophique et sur l'approche des parties prenantes et, d'autre part, d'une tournure radicale, fondée sur les théories critiques, les propositions de renouvellement actuellement existantes dans la littérature sur la GSRH demeurent normatives sinon rhétoriques, difficiles à traduire en études empiriques.

Il devient alors pertinent de se demander dans quelles mesures les perspectives plus récentes dans la littérature sur la RSE, qui se sont distancées des approches traditionnelles et qui ont servi de bases à des travaux empiriques récents, peuvent être sources de renouvellement dans la littérature sur la GSRH.

\section{Revue de la littérature sur la RSE}

Trois principaux courants constituent la littérature sur la RSE des cinquante dernières années: 1) la prescription, 2) le questionnement et 3) la compréhension empirique. Comme l'illustre le tableau 1 (Les principaux courants dans la littérature sur la RSE) ${ }^{290}$, ils se distinguent les uns des autres par la finalité des travaux qui les composent ${ }^{291}$, leurs ancrages théoriques ${ }^{292}$ et leur positionnement dans le débat sur la pratique ${ }^{293}$ et dans celui sur la théorie de la $\operatorname{RSE}^{294}$.

\footnotetext{
${ }^{290}$ La présente revue de la littérature est basée sur le recensement des écrits sur la RSE et sur les thèmes connexes, dont la citoyenneté corporative et le développement durable, issus des bases de données bibliographiques traditionnellement utilisées dans la recherche en gestion (ABI/Inform Global, Emerald Management Xtra, ISI Web of Science Social Science Citation Index, JSTOR et Repère) mais aussi d'autres sources telles que des actes de conférence publiquement disponibles, des publications de centres, de chaires et de laboratoires de recherche ainsi que des synthèses de rencontres académiques. La classification que nous avons utilisée est fondée sur l'approche de Deetz (1996). Pour identifier les différents types de recherche en science des organisations, Deetz a favorisé l'approche discursive et proposé deux dimensions (l'origine des concepts et la formulation des problèmes dans la recherche : local/émergent versus élite/a priori, d'une part; et la relation de la recherche avec le discours dominant: consensus versus dissensus) pour en déduire qu'il existe quatre types de recherche: normative, dialogique, interprétative et critique.

${ }^{291}$ Il s'agit de modéliser un idéal de RSE à forte validité opérationnelle (instrumentale ou normative) pour les tenants de la prescription, de questionner les modèles théoriques dominants de RSE et de proposer un nouvel éclairage pour les tenants du questionnement, et de comprendre empiriquement le phénomène de RSE (construction, appropriation, institutionnalisation, diffusion) pour les tenants du courant de la compréhension.

${ }^{292}$ Approches managériales traditionnelles pour les travaux de la prescription; approches sociologiques et économico-politiques pour les travaux de questionnement; et approches issues d'autres siences sociales mais appliquées aux théories des organisations pour les travaux de compréhension.

${ }^{293}$ L'auto-régulation des entreprises (versus l'intervention de l'Etat) a été beaucoup prônée par les tenants de la prescription, surtout ceux d'orientation instrumentale, comme favorisant la RSE. Le droit et les autres formes de régulation institutionnelle sont incontournables, selon les tenants du questionnement, pour induire de véritables transformations. Les acteurs à l'intérieur des organisations ont, selon les tenants de la compréhension, un rôle à jouer dans la construction collective d'un principe
} 
Les travaux de la prescription, majoritairement d'origine anglo-saxonne, et qui ont dominé la littérature du milieu des années 1950 jusqu'à la fin des années 1990, ont été mus par le même objectif de proposer des modèles de RSE justifiés par des finalités instrumentales ou normatives. Ils forment encore le courant dominant et correspondent aux trois écoles identifiées par Gendron (2000), soit la Business Ethics, la Business and Society et la Social Issue Management, ainsi que les théories de la firme identifiées par McWilliams et al. (2006), soit principalement celles de l'agence et de dépendance des ressources. Que leurs propositions soient instrumentales, prônant les avantages économiques de la RSE (corporate responsibility), ou qu'elles soient normatives, stipulant sa finalité morale (social responsibility), c'est la validité opérationnelle de la recherche qui importe dans ce courant ${ }^{295}$. Ainsi, si pour les tenants des approches instrumentales, la priorité a été de proposer des outils de management responsable créateurs de valeur économique $^{296}$, notamment via un positionnement stratégique favorable ${ }^{297}$; pour ceux des approches normatives, la priorité a été de spécifier les composantes substantives et procédurales du renouvellement de la gouvernance d'entreprise ${ }^{298}$ et d'une démarche de bonne citoyenneté d'entreprise ${ }^{299}$.

de RSE qui leur est compréhensible et que les membres de chaque organisation peuvent s'approprier.

${ }^{294}$ Il s'agit de développer de nouveaux concepts (par exemple, performance sociale organisationnelle, citoyenneté d'entreprise, gouvernance partenariale, gestion de risques sociaux, éthiques et environnementaux) pour mieux comprendre la RSE voire la remplacer, pour les tenants de la prescription; de replacer la RSE dans le débat social, pour les tenants du questionnement, d'utiliser la RSE pour mieux comprendre d'autres phénomènes organisationnels qu'elle représente empiriquement (par exemple, la construction de sens, l'apprentissage, le mimétisme) ou vice-versa, pour les tenants de la compréhension.

${ }^{295}$ La validité opérationnelle est considérée comme faisant partie des critères qui définissent l'utilité pratique de la recherche, en supposant que les gestionnaires preneurs de décisions sont les principaux utilisateurs de la recherche en science des organisations (Thomas et Tymon, 1982; Shrivastava, 1987).

${ }^{296}$ Ainsi, plusieurs études ont été menées, au cours des trente dernières années, pour démontrer les impacts positifs de la performance sociale des entreprises sur leur performance financière (pour une synthèse, voir Griffin et Mahon, 1997; Carroll, 2000).

${ }^{297}$ Ceux qui ont favorisé l'utilisation de la théorie de dépendance des ressources dans l'analyse de la RSE (par exemple, Litz, 1996 ; Russo et Fouts, 1997) ont, par exemple, conceptualisé la RSE comme une ressource ou une capacité organisationnelle qui devrait être source d'avantages compétitifs durables.

${ }^{298}$ La contestation la plus marquante de la primauté des actionnaires dans la théorie traditionnelle de la gouvernance a été attribuée à Dodd dans le fameux débat qui l'opposait à Berle au début des années 1930. Dans le courant Business and Society, les travaux de Post et Preston (Preston et Post, 1974 ; Donaldson et Preston, 1995 ; Preston, 1998 ; Preston et Donaldson, 1999 ; Post et al., 2002) se sont avérés représentatifs de la réclamation d'une redéfinition du droit et du devoir des entreprises et de l'inclusion des parties prenantes autres que les actionnaires aussi bien dans la structure que dans le fonctionnement de leur gouvernance.

${ }^{299}$ Le développement théorique de ce nouveau concept s'est fait en deux temps à travers les travaux de Wood et de Logsdon (Wood et Logsdon, 2002; Logsdon et Wood, 2002; Wood, 2004). D'abord, en évaluant dans quelles mesures l'idée de la citoyenneté définie au niveau individuel avec une approche politique pourrait se transposer au niveau organisationnel, les auteures ont stipulé que les organisations, plus particulièrement les multinationales, devraient se présenter comme des acteurs communautaires légitimes, mues par les valeurs de leurs communauté. Ensuite, en considérant le fait que l'organisation 
Les travaux du courant du questionnement, ayant émergé à la fin des années 1990, ont eu comme principal objectif de se distancer des approches managériales traditionnelles, de relever les limites ce celles-ci, en constestant leurs principales hypothèses, et de proposer de nouvelles voies d'éclairage appartenant à d'autres disciplines, notamment la sociologie (théorie de la régulation, école des représentations sociales, école des nouveaux mouvements socio-économiques, théorie des conventions) et l'économie politique. Ces travaux sont majoritairement d'origines française et québécoise et se sont développés subséquemment à l'identification par Gendron (2000) de la problématique d'asymétrie entre le questionnement éthique et social de l'entreprise, soit un questionnement de niveau institutionnel, d'une part, et la réponse organisationnelle formulée dans la conceptualisation et la pratique de la RSE, d'autre $\operatorname{part}^{300}$.

Les travaux du courant de la compréhension, s'étant développés au cours des cinq dernières années, ont été fortement hétérogènes en termes théoriques, thématiques et de niveau d'analyse. Néanmoins, ils ont convergé vers un objectif similaire de comprendre la RSE soit comme un phénomène organisationnel ayant cours dans l'espace économique, social, politique ou psychologique; soit comme un phénomène économique, social, politique ou psychologique ayant cours dans l'espace organisationnel. Alimenté par les travaux des chercheurs français, québécois et scandinaves, ce courant ne vise pas principalement ni à prescrire des modèles idéaux de RSE ni à les contester. Principalement basées sur les théories institutionnelles à la fois économiques et sociologiques, mais privilégiant davantage les niveaux d'analyse organisationnel et micro-organisationnel, les tenants de ce courant ont insisté sur le caractère contextualisé et localement construit de la $\mathrm{RSE}^{301}$. Les travaux de ce courant, davantage empiriques, se sont distancés de l'approche normative traditionnelle et se sont inscrits dans la

n'est pas seulement limitée par les règles locales mais devrait aussi répondre aux normes collectives de la communauté globale, les auteures ont proposé une autre transition, celle vers une vision des droits universels. Ayant des droits et des devoirs envers les individus et les sociétés à l'intérieur des frontières culturelles et nationales ainsi qu'à travers celles-ci, les multinationales se voient attribuer le défi d'évaluer les conditions de pertinence des normes éthiques universelles (hypernorms) et de conformité aux cultures locales.

${ }^{300}$ Les écrits de ce courant ont visé à démontrer que les initiatives dites responsables entreprises par les organisations, surtout celles privées, ne peuvent pas contribuer au renouvellement institutionnel revendiqué par les autres acteurs sociaux dans la mesure où elles sont discrétionnaires et autorégulées. Il faudrait, selon eux, réfléchir davantage sur le rôle du droit et des acteurs institutionnels tels l'État (Gendron et al., 2004), sur le «potentiel régulatoire » des normes substantives fondées sur le droit international (Gendron et al., 2003), sur les « modes de régulation » portés par les nouveaux mouvements socio-économiques (NMSÉ) dont la mobilisation a permis l'émergence de pratiques d'avant-garde (Gendron, 2002; Gendron, 2005) sur la «conjonction effective » des pratiques déjà amorcées par les entreprises avec les mouvements sociaux (Gendron, 2005) et sur la portée de la consolidation d'un «système de régulation hybride » (Gendron et al., 2004).

${ }^{301}$ Ainsi, les théories cognitives (à laquelle on associe Newell et Simon, 1972 ; Daft et Weick, 1984 ; Huber, 1991, Weick, 1995), absentes dans les deux courants précédents, y ont trouvé leur place. 
démarche récente visant à mieux comprendre le processus institutionnel amenant à la construction, à l'utilisation et au changement de la $\mathrm{RSE}^{302}$. Comme l'a précisé Boxenbaum (2006., p.46): In recent years, scholars have increasingly abandoned the quest for a normative CSR theory and a universal CSR definition. Instead, they have turned their attention to the contextual features of CSR, calling for a better understanding of the institutional processes that shape the creation, utilization, and change of CSR.

Tableau 1 : Les principaux courants dans la littérature sur la RSE

\begin{tabular}{|c|c|c|c|}
\hline & $\begin{array}{c}\text { Prescription } \\
\text { (instrumentale et normative) }\end{array}$ & Questionnement & Compréhension \\
\hline 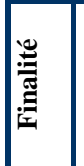 & $\begin{array}{l}\text { - Validité opérationnelle de la } \\
\text { recherche }\end{array}$ & $\begin{array}{l}\text { - Critique des approches } \\
\text { dominantes et } \\
\text { proposition d'un } \\
\text { nouvel éclairage } \\
\text { théorique }\end{array}$ & $\begin{array}{l}\text { - Éclairage empirique } \\
\text { du phénomène RSE }\end{array}$ \\
\hline 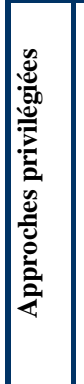 & $\begin{array}{l}\text { Approches managériales } \\
\text { traditionnelles (théories de la } \\
\text { firme, éthique utilitariste, } \\
\text { approche instrumentale des } \\
\text { parties prenantes, Issue } \\
\text { Management) } \\
\text { Approches normatives (éthique } \\
\text { normative, approche normative } \\
\text { des parties prenantes, Business } \\
\text { and Society) }\end{array}$ & $\begin{array}{l}\text { - Approches } \\
\text { sociologiques } \\
\text { (représentations } \\
\text { sociales, régulation) } \\
\text { - Économie politique }\end{array}$ & $\begin{array}{l}\text { - Théories } \\
\text { institutionnelles } \\
\text { (économiques et } \\
\text { sociologiques) } \\
\text { - Approches issues } \\
\text { d'autres sciences } \\
\text { sociales appliquées } \\
\text { aux théories des } \\
\text { organisations } \\
\text { (conventions, } \\
\text { construction sociale, } \\
\text { systèmes) }\end{array}$ \\
\hline 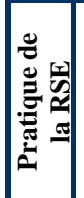 & $\begin{array}{l}\text { - Politiques publiques } \\
\text { - Avantages stratégiques } \\
\text { - Gouvernance }\end{array}$ & $\begin{array}{l}\text { - Discours des } \\
\text { entreprises } \\
\text { - Potentiel régulatoire } \\
\text { des normes, des } \\
\text { NMSE, des } \\
\text { mécanismes hybrides. }\end{array}$ & $\begin{array}{l}\text { - Compréhension } \\
\text { - Appropriation } \\
\text { - Utilisation } \\
\text { - Rôle des individus }\end{array}$ \\
\hline 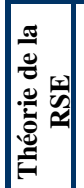 & $\begin{array}{l}\text { Définitions et dimensions de la } \\
\text { RSE } \\
\text { - Développement de concepts } \\
\text { connexes }\end{array}$ & $\begin{array}{l}\text { - La RSE dans le débat } \\
\text { social }\end{array}$ & $\begin{array}{l}\text { - Construction et } \\
\text { institutionnalisation } \\
\text { de la RSE }\end{array}$ \\
\hline
\end{tabular}

${ }^{302}$ Voir par exemple Cramer et al. (2004); Jonker et al. (2004); Boxenbaum (2006); Gond et Herrbach (2006). 


\section{Revue de la littérature sur la GSRH}

Il importe d'abord de distinguer l'approche stratégique en gestion des ressources humaines, ou ce que nous désignons ici par GSRH, de celle traditionnelle.

L'approche traditionnelle, sur laquelle se sont basés la plupart des travaux en gestion des ressources humaines au cours des années 1980, était largement axée sur l'efficacité des taches plutôt que sur le développement des humains (Analoui, 1998). Les pratiques touchant l'emploi et les relations de travail étaient étudiées individuellement, plutôt que dans leur configuration d'ensemble. Les travaux théoriques, dans l'approche traditionnelle, visaient principalement à formuler des propositions explicatives du lien direct entre ces pratiques et les mesures de la performance financière. Les travaux empiriques, quant à eux, visaient à valider de telles propositions ${ }^{303}$.

La GSRH se distingue de l'approche traditionnelle par le fait qu'elle s'intéresse à l'intégration de l'ensemble des pratiques touchant le travail et les travailleurs au processus de décision stratégique des organisations (Karami et al., 2004). Une telle intégration est conçue non seulement comme une adaptation aux changements qu'impose continuellement le contexte, notamment les changements apportés par la mondialisation et les changements technologiques (Beer, 1997), mais aussi comme une démarche de création d'avantages concurrentiels (Miller, 1989).

Bien que le concept de GSRH ait existé dans la littérature dès le début des années $1980^{304}$, ce n'est qu'au cours des années 1990 que sa formulation théorique s'est davantage précisée avec l'utilisation de la théorie de dépendance des ressources comme principal ancrage. Initialement formulée par Penrose (1959) et renouvelée notamment par Barney (1991), la théorie de dépendance des ressources suppose que les ressources qui sont rares et valeureuses sont à l'origine d'avantage concurrentiel, et qu'un tel avantage perdure si ces ressources sont protégées contre l'imitation, le transfert et la substituabilité. Dans la littérature sur la GSRH, son application a résulté en une focalisation sur le processus de développement de capacités (voir par exemple Boxall, 1996; Lundy, 1994; Mabey et al., 1998) plutôt que sur la seule analyse de la corrélation entre les activités de gestion des ressources humaines et les mesures de la performance financière. La GSRH s'est trouvée ainsi conçue comme une source clé d'avantage concurrentiel durable

\footnotetext{
${ }^{303}$ Par exemple, Delery et Doty (1996) ont stipulé que les modes universalistes, de contingence et configurationnelles de théorisation de la gestion des ressources humaines prédisent l'impact positif des pratiques (promotion interne, formation, reconnaissance, participation aux bénéfices, sécurité d'emploi, dialogue, bonne définition de tâches) sur la performance financière de la firme. Une étude empirique sur un peu plus d'un millier de banques a été ensuite menée pour valider ces propositions.

${ }^{304}$ On attribue à Fombrun et al. (1984) l'introduction initiale de ce concept.
} 
lorsqu'elle se traduit notamment par les compétences et l'engagement des travailleurs, la gestion par des spécialistes et l'intégration de la gestion des ressources humaines à la stratégie d'entreprise (Mabey et al., ibid.). La préoccupation pour la performance s'est encore plus accentuée, mais plutôt que de stipuler l'existence d'un impact direct des activités de gestion des ressources humaines sur la performance, les tenants de l'approche stratégique ont favorisé une explication de ce lien par l'existence de concepts ou variables médiateurs ${ }^{305}$.

Ce sont ensuite ces variables qui influencent favorablement la performance. La visée des travaux fondés sur l'approche stratégique devrait alors être l'étude du processus par lequel les systèmes de gestion des ressources humaines influencent les principales variables intermédiaires qui affectent la performance en termes de création de valeur actionnariale (Youndt et al., 1996; Becker et al., 1997). La littérature sur le capital intellectuel (Bontis, 1996; Edvinsson et Malone, 1997; Stewart, 1997; Nahapiet et Ghoshal, 1998) a été particulièrement mise à profit pour enrichir une telle perspective. Au cours des années 2000, c'est la thématique de la gestion durable des ressources humaines (sustainable human resource management) qui semble avoir suscité l'intérêt des tenants de la GSRH. L'utilisation des concepts de triple reddition de compte (triple bottom line) ${ }^{306}$ et de RSE a été récurrente dans les travaux ayant traité de la gestion durable des ressources humaines. La conceptualisation de la RSE qui s'est exprimée à travers ceux-ci est proche de celle favorisée par les approches managériales traditionnelles, notamment la Issue Management. Le fondement théorique de ces travaux n'a pas connu de renouvellement et est demeuré la théorie de dépendance des ressources, où la gestion durable des ressources humaines est conçue comme moyen de création de valeur lorsqu'elle est adéquatement intégrée à la poursuite de la durabilité organisationnelle ${ }^{307}$. Ces travaux (par exemple, Colbert et Kuruez, $2007^{308}$; Wirtenberg et al. $2007^{309}$ ) se sont donc principalement intéressés au rôle

\footnotetext{
${ }^{305}$ Par exemple, Youndt et Snell (2004) ont stipulé que les pratiques de gestion des ressources humaines n'accroissent pas directement la performance organisationnelle, mais qu'elles permettent d'améliorer les connaissances et le savoir des employés (capital humain), de faciliter l'interaction des groupes et le partage de connaissances (capital social) et de permettre à l'organisation d'accumuler des connaissances en systèmes, routines, processus et cultures (capital organisationnel).

${ }^{306}$ Dans sa définition la plus simple, la triple bottom line inclut, selon Elkington (1994 et 1997), les considérations liées non seulement à la création de valeur économique par les entreprises mais aussi celles liées aux valeurs sociale et environnementale qu'elles ajoutent ou qu'elles détruisent.

${ }^{307}$ La durabilité organisationnelle a été présentée par les tenants de la théorie de la dépendance des ressources comme le "pourquoi », soit le principal justificatif des considérations des enjeux sociaux et environnementaux dans la prise de décisions organisationnelles. Les travaux empiriques ayant appuyé cette argumentation (par exemple, Hill, 2001; Oliver, 2001) ont visé à démontrer que la poursuite de la durabilité, pour les entreprises, consiste en la création et la pérennisation de la richesse économique tout en protégeant et en améliorant l'environnement et l'équité sociale.

${ }^{308}$ Colbert et Kuruez (2007) se sont questionnés sur le rôle de la fonction RH dans la quête de durabilité organisationnelle. Bien que les résultats de leur étude menée dans trois entreprises canadiennes indiquent trois conceptions distinctes de la durabilité, celles-ci se rejoignent notamment sur sa finalité,
} 
de la fonction ressources humaines dans la poursuite de la durabilité organisationnelle.

A la lumière de la revue de littérature sur la GSRH que nous venons de faire, il apparaît que les approches traditionnelles, notamment la théorie de dépendance des ressources, demeurent prédominantes. Malgré l'introduction des concepts comme la durabilité, la triple reddition de compte et la RSE, qui imposent l'intégration des considérations sociales dont celles touchant les travailleurs dans les activités économiques des firmes, la finalité qui semble largement s'en dégager demeure instrumentale, soit le développement de compétences difficiles à imiter, à transférer et à substituer et créatrices d'avantages concurrentiels durables, comme le commande la théorie de dépendance des ressources. Il faut toutefois noter l'existence d'une contestation des approches dominantes en GSRH, bien que les écrits qu'on y associe soient encore peu nombreux. Des tentatives de tournure éthique (ethical shift) et de tournure radicale (radical shift) se sont amorcées depuis la fin des années 1980.

Les tentatives de tournure éthique ont été alimentées par les écrits fondés sur l'éthique philosophique, dont celle kantienne et rawlsienne, et sur la théorie des parties prenantes (par exemple Miller, 1996; Legge, 1996; Winstanley et al., 1996; Greenwood, 2002; Simmons, 2003). L'intérêt pour une telle tournure a émergé initialement du questionnement des pratiques menaçant le traitement juste des travailleurs telles les contrats contingents, la délocalisation et la sous-traitance qui augmentent les risques et l'insécurité, ainsi que le contrôle bureaucratique de la performance qui réduit l'autonomie (Winstanley et al., ibid.). Parmi les intérêts plus récents des tenants de la perspective éthique figure l'utilisation d'une combinaison des approches instrumentale et normative des parties prenantes ${ }^{310}$,

soit la recherche de la performance par un positionnement stratégique favorable, et sur le rôle crucial des acteurs et des processus appartenant à la fonction ressources humaines (développement organisationnel, gestion du changement, planification stratégique des ressources humaines, embauche, gestion des compétences, formation et développement professionnel) dans la poursuite d'une performance durable.

${ }^{309}$ Wirtenberg et al. (2007) se sont basés sur neuf cas pour identifier les principales qualités des entreprises les plus durables et les principales contributions actuelles de la fonction ressources humaines à la durabilité (développement de leadership, formation et développement professionnel, gestion de la diversité, éthique et gouvernance).

${ }^{310}$ Dans sa version instrumentale, l'approche des parties prenantes favorise la prise en compte des attentes des Stakeholders primaires, soit un comportement qui devrait influencer favorablement la performance organisationnelle mesurée par les indicateurs traditionnels tels la profitabilité (Steurer et al., 2005). Dans le travail fondateur de la théorie des parties prenantes, Freeman (1984) a défini le Stakeholder comme tout individu ou tout groupe qui affecte la poursuite des objectifs de l'entreprise ou qui en est affecté. Suivant la classification de Mitchell et al. (1997) selon les dimensions pouvoir, légitimité et urgence, les Stakeholders primaires sont ceux dont la survie et la continuité même de l'entreprise dépend. Dans sa version normative, l'approche des parties prenantes stipule que les Stakeholders se définissent par les intérêts légitimes qu'ils ont dans l'entreprise et qui ont une valeur 
soit à la fois conséquentialiste et déontologique, pour traiter des thématiques de la performance, de l'imputabilité et de l'équité dans les organisations. Cette perspective n'évacue pas l'impératif de rentabilité, mais contrairement au courant dit dur de la GSRH ${ }^{311}$, elle stipule que les ressources humaines ne devraient pas être utilisées de la même manière que les autres facteurs de production pour maximiser la valeur et les avantages concurrentiels, et que les preneurs des décisions stratégiques devraient reconnaître l'existence de relations équitables et négociées entre l'organisation et ses parties prenantes, dont les travailleurs. Les pratiques responsables liées aux ressources humaines, basées sur les principes d'imputabilité et de justice, sont jugées, dans ce courant comme compatibles avec la recherche de la performance. Le principe de justice envers les travailleurs mis de l'avant dans cette perspective inclut à la fois la justice redistributrice, soit l'équité dans la distribution des ressources organisationnelles, et la justice procédurale, soit l'existence d'un processus équitable de prise de décision (Andrews et Kacmar, 2001) (12 $^{312}$.

Les tentatives de tournure radicale ont été nourries, quant à elles, par les écrits favorisant les approches critiques (Critical Management Theories) qui considèrent la GSRH comme rhétorique, manipulatrice et de surcroît, comme un moyen de contrôler les travailleurs (par exemple Guest, 1987, 1997, 1999; Keenoy, 1990a, 1990b; Townley, 1993; Sennet, 1999). Plutôt que d'être un moyen de développement personnel pour les travailleurs, les pratiques de GSRH ne sont, selon les tenants de ce courant qu'une manière d'envahir la vie personnelle des travailleurs pour amener ceux-ci à contribuer encore davantage à l'atteinte des objectifs économiques organisationnels (Greenwood, 2002). Un des exemples les plus significatifs de ce type d'analyse est le travail de Townley, fondé sur les concepts d'examen et de confession de Michel Foucault ${ }^{313}$, et qui stipule que

intrinsèque méritant une considération de la part des gestionnaires (Donaldson et Preston, ibid.). L'imputabilité des entreprises devrait alors ne plus se limiter aux stakeholders primaires, définis par Mitchell et al. (ibid.) comme ceux dont leur survie dépend, ou à ceux contractuels mais étendue aux autres parties prenantes (Reed, 1999).

${ }^{311}$ Les qualificatifs 'hard' et 'soft' ont été utilisés dans la littérature en GSRH pour distinguer l'approche focalisée sur la performance financière, et considérant les ressources humaines comme moyens similaires à tous les autres types de ressources utilisés par l'organisation, de celle focalisée sur le traitement équitable des travailleurs dans le cadre de la poursuite des objectifs organisationnels. Pour les tenants de l'approche dure, il importe prioritairement de spécifier et de mesurer les contributions des travailleurs aux objectifs stratégiques (Armstrong et Baron, 2000). Pour les tenants de l'approche molle, les travailleurs devraient être considérés comme des humains dotés de ressources (resourceful humans) plutôt que comme des ressources humaines (human resources) (Simmons, ibid.).

${ }^{312}$ Par exemple, une étude de Lemons et Jones (2000) indique que les travailleurs qui se considèrent traités équitablement par leurs employeurs sont plus impliqués et satisfaits au travail. Pettijohn et al. (2001) stipulent l'existence d'une relation négative entre le traitement équitable des travailleurs et le roulement du personnel.

${ }^{313}$ Selon Foucault (cité par Townley, ibid.), les processus d'examen et de confession permettent de connaître l'individu. L'examen consiste à l'observer afin de l'évaluer et de le classer. La confession est 
l'individu est mesuré et évalué par l'entreprise pour le rendre calculable et gérable. Dans la même veine, Sennet décrit l'entreprise moderne comme un réseau d'équipes semi-autonomes en roulement continu, dans lequel les ressources humaines sont allouées selon la demande du marché, le pouvoir est centralisé entre les mains d'une élite managériale et la relation de travail est purement contractuelle. Le courant critique questionne voire conteste non seulement les approches traditionnelle et stratégique de théorisation de la GSRH mais aussi celle éthique. L'approche molle incluant la gestion éthique des ressources humaines n'est, selon les tenants du courant critique, qu'une forme déguisée de l'approche dure et l'utilisation de la théorie des parties prenantes en GSRH est dénuée de moralité (Hart, 1993, cité par Simmons, ibid.) ${ }^{314}$. Même dans sa forme normative, invoquant notamment les concepts de justice et de droit, l'approche éthique obscurcit les questions incontournables de l'approche critique sur les attributs idéologique et politique de la GSRH (Barrett, 1999).

\section{Utiliser les connaissances sur la RSE pour repenser la GSRH ?}

Il est clair que dans son état actuel, la littérature sur la GSRH n'est pas suffisamment outillée pour avancer les connaissances sur la gestion des ressources humaines qui tient compte du questionnement éthique et social porté par la RSE. Les approches traditionnelles demeurent prédominantes. Les travaux basés sur celles-ci, caractérisés par la centralité des concepts tels la durabilité et les capacités (firms capabilities), soit des notions issues de la littérature stratégique, plus précisément de la théorie de dépendance des ressources, sont fondés sur la conviction que comme les ressources humaines constituent un facteur clé dans la capacité d'une organisation à créer et à pérenniser son avantage stratégique et comme la poursuite de la durabilité notamment par l'intégration des considérations économiques, sociales et environnementales à la gestion amène un tel avantage, les activités de la fonction ressources humaines devraient être menées de manière à ce qu'elles puissent contribuer à cet objectif stratégique devant se traduire en performance financière. Comme le caractérise Greenwood (ibid., p. 262) :

(...) it tends to be US-based (Legge, 2000) and practitioner focused, its focus is prescriptive and often relies on naïve generalizations that assume the value of HRM. Much of the writings has been concerned with either the offer of practical

l'exposition par l'individu de la connaissance de soi. L'évaluation des indicateurs extérieurs de compétence, de performance et de comportement au travail constitue un processus d'examen. Les procédures amenant le travailleur à produire de l'information sur soi, notamment par les sondages et l'évaluation personnelle, sont quant à elles une forme de confession.

314 «If the "hard" variant, with its emphasis on the strategic integration of human resource policy with business strategy is the "iron fist", "soft" HRM with its core motifs of commitment and capability is no more than the "iron fist in the velvet glove" (Hart, 1993, p. 29). HRM in either of its guises contains the implicit assumption that "we should behave towards our fellow human beings with the overriding objective of extracting added value" (Hart, 1993, p. 29). » (Barrett, 1999, p.309) 
advice or the presentation of empirical data (Wright and McMahan, 1992). It takes a system maintenance or a functionalist approach, viewing HRM as a mechanism for the attainment of organisational goals, and thus reflect concerns with improvement in efficiency that derive from classical management theory (Townley, 1993).

Cette critique semble fortement similaire à celle posée par les tenants du courant du questionnement, au début des années 2000, dans la littérature sur la RSE. Mais dans cette dernière, le renouvellement exhorté s'est manifesté de manière accélérée et les travaux empiriques issus des courants du questionnement et de la compréhension livrent déjà leurs résultats. Ainsi, il a été possible de démontrer que les approches prescriptives à elles seules sont limitées pour mieux comprendre la RSE. La tournure éthique en GSRH, en tentant ces dernières années de concilier les visées instrumentale et normative, n'a pas permis d'induire un renouvellement substantiel. L'ancrage de cette perspective dans la logique de compatibilité entre l'éthique et la performance la dilue dans le courant Issue Management, qui prône que 'Good ethics is good business' et que la pérennité d'une organisation passe par la prise en compte des considérations éthiques, dont celles liées aux ressources humaines.

Les questions éthiques touchant l'emploi et les travailleurs sont alors conçues comme des enjeux ou des risques à gérer afin de satisfaire les objectifs stratégiques à long terme. La préoccupation pour la performance demeure centrale dans ce courant, mais celle-ci se conçoit dans un horizon temporel plus étendu. En ce sens, la satisfaction des travailleurs se sentant traités équitablement peut être considérée comme la variable médiatrice dans la relation entre la gestion éthique des ressources humaines et la performance à long terme, bien qu'une telle explication n'ait pas été explicitement exprimée ainsi par les tenants de la théorie des parties prenantes appliquée à la GSRH. Parmi les limites de l'utilisation actuelle de l'approche éthique dans l'analyse de la GSRH selon Greenwood (ibid.): les convictions sous-tendant les théories utilisées ne sont pas explicites, alors qu'il existe différentes théories qui se réclament de l'éthique (notamment l'utilitarisme et le kantianisme); l'application des théories est souvent simpliste; le rôle de la théorie des parties prenantes n'a pas été mis à profit; et les propositions théoriques avancées sont difficiles à mettre en œuvre en pratique. L'approche radicale semble s'être fixée, quant à elle, dans une rhétorique stérile et difficile à opérationnaliser en recherche.

Parmi les trois courants identifiés dans notre revue de la littérature sur la RSE, ce sont ceux du questionnement et de la compréhension empirique qui pourraient offrir un potentiel de renouvellement de la littérature sur la GSRH. Les fondements sociologiques et économico-politiques, favorisés par des tenants du courant du questionnement, pourraient être utilisés pour argumenter qu'en pratique le 
renouvellement de la GSRH passe impérativement par le questionnement de la finalité des activités de production auxquelles participe l'humain et de la place que celui-ci y occupe. Le droit y est incontournable comme mécanisme de transformation et en théorie, repenser la GSRH nécessite le recours à des approches telles celles issues de la théorie de la régulation, se focalisant sur les questions de rapport salarial (voir par exemple, Bardelli, 2006). Les fondements institutionnels, dont ceux issus des théories cognitives, favorisés par les tenants du courant de la compréhension pourraient, quant à eux, être mis à profit pour répondre à des questions telles que comment se construisent collectivement et s'institutionnalisent les pratiques responsables de GSRH? Quelles conditions institutionnelles (par exemple, micro-mécanismes organisationnels versus transformations macroéconomiques et sociales ou une hybridation de ces deux types) sont susceptibles de générer de nouveaux construits au niveau du cadre cognitif des acteurs organisationnels concernant les enjeux touchant le travail? (voir par exemple, Boxenbaum, 2006)

\section{LES RESSOURCES HUMAINES DANS LES RAPPORTS RSE DES BANQUES CANADIENNES}

La publication d'information sur la RSE est devenue, ces vingt dernières années, une pratique adoptée par de plus en plus d'entreprises ${ }^{315}$. Plusieurs appellations ont été utilisées pour désigner le document dédié à la RSE: bilan social, rapport sociétal, rapport RSE (CSR report), rapport sur la citoyenneté corporative (Corporate Citizenship Report), rapport de développement durable, etc. Selon le pays et le secteur d'activités des entreprises, la publication d'information sur la RSE relève soit de la conformité légale soit du volontariat des entreprises. Au Canada, les institutions de dépôt ayant un actif d'au moins un milliard de dollars sont soumises, depuis 2002, au Règlement sur la déclaration annuelle qui les oblige à produire une déclaration sous forme de rapport, faisant état de leurs contributions à la société. Le présent article se concentre sur les résultats de l'analyse du contenu des rapports des six plus grandes banques canadiennes, publiés en 2002 et 2003, soit un total de 12 rapports $^{316}$, et plus spécifiquement sur

\footnotetext{
${ }^{315} \mathrm{Au}$ début, cette pratique consistait en l'inclusion de données environnementales dans les rapports financiers traditionnels, surtout lorsque ce type d'informations comportait des incidences comptables. Par la suite, on a pu observer le développement des rapports environnementaux sous la forme de documents indépendants du rapport annuel et consacrés uniquement à rendre compte de la performance environnementale. La fin des années 1990 a donné lieu à la multiplication des rapports dits « dédiés » à la RSE, à l'intérieur desquels on retrouvait des informations sur la responsabilité des entreprises envers leurs clients, leurs employés (gestion des ressources humaines) et la communauté (contributions philanthropiques) en plus de l'inclusion de données sur la responsabilité environnementale.

${ }^{316}$ Nous tenons à préciser que quatre banques étrangères jugées leaders, d'origines britannique, française, américaine et néerlandaise, ont été aussi incluses dans notre étude. Néamoins, nous avons choisi de nous concentrer ici sur le cas des six plus grandes canadiennes, qui, bénéficiant d'une situation oligopolistique, sont représentatives du secteur bancaire au Canada. Elles évoluent au sein du même
} 
l'analyse de leur discours sur la thématique de l'emploi et des employés. Avant l'année 2002, trois d'entre elles avaient déjà publié un rapport RSE, au moins une fois $^{317}$. Cependant, à compter de cette année 2002, elles ont toutes été soumises à l'obligation de communiquer une déclaration annuelle à leurs clients et au public, d'informer ceux-ci, notamment au moyen d'annonces publicitaires, de communiqués, d'affiches ou d'envois postaux, des façons dont ils peuvent se procurer la déclaration, et de leur en remettre une copie sans frais, si la demande en est faite. Outre les renseignements à caractère économique (par exemple, contributions fiscales, financement des entreprises) et communautaire (par exemple, bénévolat des employés, contributions philanthropiques), les renseignements requis concernant spécifiquement les ressources humaines incluent le nombre de personnes employées par le déclarant et les entités de son groupe qui font l'objet de la déclaration à la fin de l'exercice dans chaque province, y compris : (i) le nombre de postes à temps plein occupés par ces personnes, (ii) le nombre de postes à temps partiel occupés par ces personnes. Les banques demeurent libres d'aller au-delà du minimum requis.

L'analyse du contenu des rapports RSE est une des techniques fréquemment utilisées pour étudier les représentations de la RSE chez les entreprises et celles de leur performance à cet égard, pour repérer les pratiques jugées responsables, et de surcroît, pour évaluer leur appropriation de ce principe. Elle s'effectue généralement en utilisant la technique de codification. Cette méthode consiste à catégoriser le contenu textuel des rapports en utilisant des thèmes prédéterminés, émergents ou une combinaison de ces deux types de codes. Si certaines études ont privilégié l'analyse thématique ${ }^{318}$, nous avons opté, à l'aide du logiciel Atlas Ti, pour la triple codification ${ }^{319}$ suggérée par les tenants de la théorisation ancrée ${ }^{320}$.

cadre institutionnel et sont toutes soumises au Règlement sur la déclaration annuelle de 2002. Pour ces six banques, la déclaration annuelle obligatoire est intégrée à leur rapport RSE, bien que l'appellation utilisée varie d'une banque à l'autre (par exemple, bilan social, rapport de responsabilité publique, rapport sur les responsabilités).

${ }^{317}$ Parmi les six banques, une avait déjà publié en premier ce genre de document dès 1999. Deux autres ont emboîté le pas en publiant leur premier rapport en 2001. Ces trois institutions peuvent donc être considérées comme les précurseurs de la publication de rapports dédiés à la RSE puisqu'elles ont adopté cette pratique avant même d'y être contraintes par obligation légale. La pratique s'est ensuite généralisée.

${ }^{318}$ Il s'agit de se doter d'une liste de thèmes prédéfinis et de repérer dans les rapports la présence ou l'absence de ceux-ci (voir par exemple, Vuontisjarvi, 2006).

${ }^{319}$ Dans le détail, la démarche de codification ouverte consiste à caractériser des segments de textes en fonction de codes émergents pour la plupart, donc dégagés du texte sur un mode intuitif, mais également construits à partir du cadre théorique. La codification axiale consiste à analyser la fréquence des codes ainsi que les relations existant entre les codes, ce qui permet d'esquisser une représentation schématique de la représentation sociale d'un thème donné pour un acteur précis (chaque unité herméneutique comprend les deux rapports 2002 et 2003 d'une institution bancaire). Enfin, l'analyse transversale se fait en fusionnant les unités herméneutiques créées dans un premier temps sur une base organisationnelle, et en effectuant des opérations d'analyse sélective, c'est-à-dire en isolant les codes 
L'analyse s'est ainsi basée sur le volume d'information associé aux concepts clés de notre étude et sur les articulations entre ceux-ci.

Bien que l'étude que nous avons menée ait touché plusieurs thématiques, nous nous concentrerons ici sur les résultats de l'analyse des codes dans la thématique emplois et employés. Les banques seront désignées par BC1, BC2, BC3, BC4, BC5 et BC6.

\section{La responsabilité envers les employés comme dimension incontournable de la RSE}

La question de l'emploi et des employés constitue une des principales dimensions de la RSE des banques canadiennes. D'une part, l'analyse du code «Vision $\mathrm{RSE} »^{321}$ a révélé que les employés sont explicitement identifiés par les banques comme faisant partie des principaux partenaires envers lesquels elles se considèrent responsables ${ }^{322}$. D'autre part, comme l'indique le tableau 2 (voir Tableau 2: Les codes les plus fréquents dans les rapports RSE des banques canadiennes), les principales préoccupations que les banques relèvent dans leurs rapports incluent celles touchant l'emploi et les employés (code «Responsabilité

relatifs à une question de recherche dans une famille, qui fait ensuite l'objet d'une codification axiale. La famille analysée dans le cadre du présent article s'intitule "Emploi et employés », et regroupe tous les codes relatifs à ce thème. Notons que nous avons procédé dans un premier temps à une analyse de contenu thématique et qualitative, alors que l'analyse transversale repose sur une analyse de contenu quantitative, triangulée avec les résultats antérieurs de la codification axiale de chaque unité herméneutique organisationnelle.

320 « The aim (of grounded theory methodology) is to discover what kinds of concepts and hypotheses are relevant to the area one wishes to understand. Grounded theory, therefore, provides new insights into the understanding of social processes emerging from the context in which they occur, without forcing and adjusting the data to previous theoretical frameworks. »(Glaser, 1995, 1998 cité dans Lansisalmi et al., 2004, p. 242).

${ }^{321}$ Le code «Vision RSE » $(\mathrm{n}=50)$ a été utilisé pour les passages (citations) dans lesquels les banques expriment leur définition ou leur conception de ce principe, ou dans lesquels elles énoncent leur politique à l'égard de la RSE, incluant leus objectifs et ce qu'elles considèrent comme leurs principales réalisations. Ces passages se trouvent généralement dans la partie introductive des rapports, notamment dans le message de la direction ou dans les extraits de témoignage des employés et des cadres. Le code «Vision RSE » ne fait pas partie des codes les plus fréquents. Néanmoins, comme l'une de nos questions de recherche était de connaître la compréhension que les banques ont de ce principe, il était impératif d'analyser ce code en profondeur.

${ }^{322}$ Les termes favorisés par les banques canadiennes pour déclarer leur RSE varient d'une banque à l'autre ( «contribution à la cohésion sociale», «citoyenneté corporative », « responsabilités » au pluriel). Néanmoins, malgré un vocabulaire hétérogène, les relations avec les parties prenantes sont au cœur de la définition de la RSE. Bien que les appellations utilisées pour désigner ces parties qui affectent et qui sont affectés par les activités des banques varient (par exemple, «intervenants », « intéressés », «partenaires »), l'identification des suivantes est récurrente: les clients, les employés, les collectivités, les actionnaires et l'environnement. La considération des attentes de ces parties pranantes définit principalement ce que les banques entendent par RSE. 
envers les employés » ${ }^{323}$ ).

Comme type de responsabilité, celle envers les employés se trouve au troisième rang en importance chez les banques, soit après celle envers la communauté et celle envers les clients, mais avant celle envers les actionnaires et celle envers l'environnement. Ce portrait, qui reflète une forte priorisation des questions touchant les membres de la communauté $e^{324}$, les clients et les employés, est commun à la grande majorité des six banques ${ }^{325}$. La responsabilité envers les employés est jugée comme incontournable dans la définition de ce qu'est une banque responsable ou citoyenne ${ }^{326}$, et plus spécifiquement, dans la mesure ou une telle responsabilité ou une telle citoyenneté est considérée comme compatible avec la poursuite d'avantages stratégiques ${ }^{327}$.

Une des questions les plus récurrentes dans le débat sur la pratique de la RSE est celle de savoir si ce principe est compatible avec la poursuite de la rentabilité de l'entreprise. La vision véhiculée par les banques canadiennes dans leurs rapports semble y répondre de manière affirmative. Ainsi, elles considèrent que le comportement responsable est compatible avec la recherche de la pérennité organisationnelle que celles-ci désignent par «développement durable d'une entreprise », « résultats financiers durables », «viabilité à long terme ${ }^{328}$. Les

${ }^{323}$ Le code « Responsabilité envers les employés » $(\mathrm{n}=203)$ a été utilisé pour les passages (citations)
traitant de toute question touchant l'emploi et les employés, qu'il s'agisse de vision, de politiques, de
systèmes, de procédures, de législations, de normes, de codes ou chartes internes, d'activités de gestion
des ressources humaines, de statistiques sur l'emploi et sur les employés, etc.
${ }^{324}$ La prédominance du traitement des questions touchant les membres de la communauté dans les
rapports des banques se reflète non seulement par la fréquence du code « Responsabilité envers la
communauté » $(\mathrm{n}=571)$ mais aussi par la fréquence respective des codes suivants appartenant à la
thématique de l'engagement philanthropique : «Dons et commandites » $(\mathrm{n}=391)$, «Partenariat avec
OBNL » $(\mathrm{n}=230)$, « Bénévolat employés et cadres » $(\mathrm{n}=227)$, et «Éducation dans communauté » $(\mathrm{n}=$ 220).

${ }^{325}$ A l'exception de $\mathrm{BC} 3$, où l'importance attribuée aux préoccupations environnementales semble dépasser celles touchant les employés, $(n=46$ pour le code "Responsabilité envers l'environnement » versus $n=33$ pour le code « Responsabilité envers les employés »), toutes les banques étudiées placent la responsabilité envers les employés au troisième rang après celle envers la communauté et celle envers les clients.

326 «La 'responsabilité d'une entreprise' et le rôle d'une 'entreprise citoyenne' se définissent de plus d'une manière. Mais chose certaine, une société ne peut pas se contenter d'envoyer un chèque à des organismes caritatifs et croire sa mission accomplie. Elle a aussi une responsabilité envers ses employés, ses clients et ses actionnaires ainsi qu'envers ses collectivités. » (BC1)

327 « À la BC5, notre objectif premier est d'être le meilleur et le plus prospère des groupes canadiens de services financiers à vocation internationale. Nous avons toujours entendu le terme « succès » au sens large, de sorte à englober et à équilibrer les intérêts variés de nos différents partenaires. Offrir un service à la clientèle supérieur, consolider notre réputation en tant qu'employeur de premier ordre et contribuer à la santé globale et au bien-être de nos collectivités, voilà autant d'éléments importants par lesquels nous mesurons notre succès - sans oublier, bien entendu, créer une valeur sûre et à long terme pour nos actionnaires. ». (BC5)

328 « Le développement durable d'une entreprise consiste à créer de la valeur à long terme pour les actionnaires en saisissant les occasions et en gérant les risques liés à la conjoncture économique, 
pratiques dites responsables envers les employés semblent donc s'orienter prioritairement vers la recherche du statut d' « employeur de choix », et de surcroît, vers la poursuite d'une finalité stratégique, soit celle d'un positionnement favorable et créateur de valeur.

Tableau 2 : Les codes les plus fréquents dans les rapports RSE des banques canadiennes

\begin{tabular}{|l|r|r|r|r|r|r|r|}
\hline Codes/Banques & BC1 & BC2 & BC3 & BC4 & BC5 & BC6 & Total \\
\hline Responsabilité envers la communauté & 89 & 62 & 163 & 80 & 94 & 83 & 571 \\
\hline Responsabilité envers les clients & 49 & 63 & 108 & 61 & 71 & 49 & 401 \\
\hline Dons et commandites & 76 & 34 & 94 & 60 & 70 & 57 & 391 \\
\hline Partenariat avec OBNL & 42 & 25 & 67 & 30 & 40 & 26 & 230 \\
\hline Bénévolat employés et cadres & 47 & 35 & 49 & 28 & 52 & 16 & 227 \\
\hline Éducation dans communauté & 53 & 19 & 40 & 44 & 36 & 28 & 220 \\
\hline Responsabilité envers les employés & 28 & 35 & 33 & 35 & 45 & 27 & 203 \\
\hline Réseaux de distribution et accessibilité & 22 & 26 & 54 & 36 & 30 & 21 & 189 \\
\hline Inclusion sociale/diversité client & 29 & 15 & 21 & 8 & 11 & 23 & 107 \\
\hline
\end{tabular}

\section{Quelle responsabilité envers les employés?}

Le discours des banques canadiennes relativement à la responsabilité envers les employés peut être caractérisé par l'analyse de huit codes que nous avons regroupés dans la famille «Emploi et employés » (Tableau 3).

Tableau 3 : Les codes de la famille «Emploi et employés »

\begin{tabular}{|l|l|}
\hline Responsabilité envers les employés & 203 \\
\hline Diversité main d'oeuvre/équité & 79 \\
\hline Formation et développement & 74 \\
\hline Données/ emploi et employés & 33 \\
\hline Dialogue/consultation des employés & 33 \\
\hline Rémunération et avantages & 27 \\
\hline Conciliation emploi/famille & 27 \\
\hline Santé et sécurité & 11 \\
\hline
\end{tabular}

Cette première étape de notre analyse vise à identifier les préoccupations plus spécifiques des banques relativement aux questions de l'emploi et des employés, et

environnementale et sociale. ».(BC1). 
par conséquent, de dégager la perspective dans laquelle elles envisagent les enjeux associés au monde du travail, dans leur démarche de responsabilité sociale, et conçoivent leur responsabilité envers les employés.

Outre le code « Responsabilité envers les employés » $(\mathrm{n}=203)$, qui, rappelons-le, a été utilisé pour toutes les citations traitant des questions touchant l'emploi et les employés, les deux autres codes les plus fréquents sont «Diversité main d'oeuvre/équité » $(\mathrm{n}=79)$ et «Formation et développement» $(\mathrm{n}=74)$. Leur occurrence respective, qui est de loin supérieure à celle de chacun des cinq autres codes de la famille «Emploi et employés », indique que le discours des banques canadiennes à l'égard des employés est focalisé sur les enjeux suivants:

- La promotion de la diversité à l'embauche: soit la prise en compte de la diversité de la main-d'œuvre (par exemple, en termes d'appartenance ethnique), en établissant des politiques et des pratiques de représentation des divers groupes de la communauté au niveau de l'effectif. Il s'agit aussi de favoriser l'embauche des autres groupes traditionnellement défavorisés comme les femmes et les membres de la communauté autochtone ${ }^{329}$. En ce sens, le principe de diversité est sous-tendu par celui de l'inclusion.

- Le traitement équitable des employés sans égard à leurs différentes appartenances sociales: l'idée d'inclusion, dans le sens d'anti-discrimination, sous-tend aussi celle d'équité, va au-delà des initiatives visant l'embauche et implique l'accommodement des employés ayant des besoins spécifiques (par exemple, l'aménagement physique des lieux de travail pour les employés à mobilité réduite ${ }^{330}$ ). Ainsi, le chevauchement des questions de diversité et d'équité ${ }^{331}$ est significatif.

- La formation à l'emploi : qui met à la disposition des employés les outils d'apprentissage nécessaires à l'exercice de leur métier. Le développement professionnel, quant à lui, n'est pas nécessairement impératif dans l'immédiat mais offre aux employés l'opportunité d'apprendre et de progresser dans leur carrière ${ }^{332}$.

329 «Un des objectifs de BC1 est de recruter davantage de personnes handicapées. Grâce à des partenariats avec des organismes nationaux, comme le Conseil Canadien de la Réadaptation et du Travail, et locaux, comme Champions of Diversity à Calgary, $\mathrm{BC} 1$ place des candidats des minorités visibles ou des personnes handicapées dans des emplois et dans des postes de stagiaires ou de formation. » (BC1).

330 « Le programme Accès facile est une des pierres angulaires de notre engagement à assurer un lieu de travail adapté. Il permet aux gestionnaires d'approuver sur-le-champ des dépenses liées à l'accessibilité pouvant atteindre 3000 \$ par employé, par année. Au besoin, le Conseil Canadien de la Réadaptation et du Travail fait des évaluations sur place et donne des recommandations d'expert au gestionnaire et à l'employé. En général, nous communiquons nos politiques d'aménagement des postes de travail aux gestionnaires pour nous assurer qu'ils comprennent notre engagement. » (BC1).

${ }_{331}$ «Pour assurer l'équité en matière d'emploi à nos employés qui souffrent d'un handicap physique ou sensoriel, nous avons commencé l'adaptation de nos édifices en 1990. Une grande partie des espaces ont été réaménagés à ce jour, facilitant ainsi l'accès et le déplacement de ces employés. » (BC2).

332 «We also believe that standing behind our employees today means helping them prepare for tomorrow. They are thinking beyond their immediate work life, whether they are considering their career objectives or their changing family obligations. At BC4, we've developed a wide spectrum of 
Quatre autres codes de la famille «Emploi et employés » sont moins fréquents que les trois précédents mais sont présents dans tous les rapports des six banques pour les deux années étudiées. Le code «Données/emplois et employés 》 $(\mathrm{n}=$ 33) correspond aux citations fournissant des renseignements quantitatifs sur ce que les banques considèrent comme leur performance en matière de responsabilité envers les employés. Les données présentées sont peu nombreuses et se rapportent généralement à la répartition de l'effectif par sexe, par région et par statut (employés à temps plein, employés à temps partiel). Le code «Dialogue/consultation des employés » $(\mathrm{n}=33)$ caractérise le discours des banques sur l'importance de la communication avec leurs employés, communication qui ne se limite pas à l'acte informatif (à un sens, des banques aux employés) ${ }^{333}$ mais qui vise aussi à consulter les employés et à se concerter avec eux, notamment afin d'évaluer leur satisfaction ${ }^{334}$. Le code «Rémunération et avantages » $(\mathrm{n}=27)$ précise les politiques et les pratiques considérées par les banques comme favorisant l'offre de conditions de rémunération favorables, ne se limitant pas aux salaires et avantages sociaux mais incluant aussi la reconnaissance du mérite. Le code «Conciliation emploi/famille » $(n=27)$ indique les initiatives favorisant la conciliation de la vie professionnelle à celle familiale ${ }^{335}$ ainsi que les activités permettant ou sollicitant la participation des membres de la famille des employés ou considérées par la banque comme ayant des impacts potentiels ou réels sur ceux-ci.

Le code le moins fréquent de la famille «Emploi et employés » est «Santé et sécurité » $(\mathrm{n}=11)$. De plus, il était seulement présent pour les deux années dans les

employee services and continuous learning opportunities so our people can thrive professionally and personally today, and plan forthe possibilities that lie ahead. » (BC4)

${ }_{333}$ « La BC5 croit fermement qu'il est important non seulement de tenir ses employés au courant de tout ce qui a trait à leur emploi, mais également de recueillir leurs opinions et points de vue. Nous encourageons la communication bilatérale : nous voulons que les employés comprennent bien nos buts et stratégies, qu'ils aient facilement accès à l'information quotidienne touchant leur emploi à la BC5, leur rendement, les avantages sociaux et les services, et qu'ils aient l'occasion d'exprimer franchement leurs vues et leurs préférences. » (BC5)

334 «C'est la priorité de notre entreprise de traiter ses employés avec le même respect qu'ils démontrent envers les clients. En 2003, le Service des ressources humaines lançait un projet directeur appelé Amélioration des processus et changement dans la concertation (IMPACT) pour discerner et contrer les obstacles au bien-être du personnel. Des groupes de consultation pancanadiens formés d'employés, de gestionnaires et de spécialistes des ressources humaines ont dressé une liste de 200 obstacles allant des salaires accordés pour un travail intense aux politiques vagues sur les avantages sociaux. Des projets à court, moyen et plus long terme sont en voie de mise au point pour régler ces problèmes, et nos progrès en ce sens sont régulièrement annoncés sur le site intranet des employés et par courriel. » (BC4)

335 «Pour beaucoup, le travail constitue le moyen de fonder une famille et d'en assumer la responsabilité. Afin d'aider nos employés à concilier travail et vie personnelle, nous avons consenti d'importants efforts qui se sont d'abord traduits, dès 1980, par l'ouverture de garderies en milieu de travail, puis dans le développement d'une politique de congés familiaux qui s'ajoute à la politique de congés parentaux légaux. » (BC2) 
rapports de trois banques. Ce code correspond aux citations qui rendent compte des activités entreprises par les banques pour assurer à leur personnel un environnement de travail sain et sécuritaire, qui semble ne pas se limiter aux aménagements physiques favorables des lieux de travail mais s'élargit à des programmes de soin de santé incluant la prévention, le conseil et le support (par exemple, l'aide psychologique et la formation sur le SIDA).

\section{Quel potentiel de changements?}

Cette deuxième étape de notre analyse vise à évaluer si les pratiques dominantes actuelles qu'on associe à la responsabilité sociale ainsi que les indicateurs utilisés pour en mesurer les résultats sont précurseurs de véritables transformations dans les relations des banques avec leurs employés. Une telle évaluation s'effectuera en procédant à une analyse plus fine des trois codes les plus fréquents, soit «Responsabilité envers les employés » $(\mathrm{n}=203)$, « Diversité main d'œuvre/ équité $»(\mathrm{n}=79)$ et «Formation et développement » $(\mathrm{n}=74)$. En tentant d'identifier le cadre «moyen/fin » (mean/end frame) dominant qui se dégage du discours des banques canadiennes, nous serons en mesure de déduire s'il y a ou non un potentiel de renouvellement des pratiques et de la manière d'en mesurer les résultats pour répondre à l'impératif de passage vers un véritable développement social des employés. Le cadre «moyen/fin » est une représentation utilisée dans les approches cognitives pour caractériser le lien entre un construit et sa finalité (Boxenbaum, ibid.). Ici, nous visons à déterminer si le cadre «moyen/fin » dominant tend vers une logique d'efficience minimaliste ${ }^{336}$, une logique de positionnement stratégique ${ }^{337}$ ou une logique d'imputabilité sociale ${ }^{338}$.

\footnotetext{
${ }^{336} \mathrm{La}$ logique d'efficience minimaliste s'exprimerait par un discours focalisé sur la performance financière. Le message typique qui s'en dégage serait: «We are a successful company with a welleducated and highly competent workforce. » (Vuontisjarvi, ibid., p. 345).

${ }^{337} \mathrm{La}$ logique de positionnement stratégique se traduirait par un discours qui nuance la performance, en étendant son horizon à un plus long terme. Les initiatives de responsabilité envers les employés seraient considérées comme un investissement visant à se constituer un capital intellectuel. Le message typique qui s'en dégagerait serait : «We are a socially responsible company, taking good care of our employees. (...) a well-educated personnel in a good state of health contributes to the corporate economic performance » (Vuontisjarvi, ibid., p. 345).

${ }^{338}$ La logique d'imputabilité sociale (social accountability) est caractérisée par le questionnement profond des pratiques actuelles afin de répondre aux appels continuels à l'imputabilité envers la société, offreuse de main-d'œuvre aux entreprises (versus seule imputabilité aux actionnaires). Cette logique transcende l'approche focalisée sur la durabilité économique des entreprises et cible, comme finalité, le développement social des employés (Vuontisjarvi, ibid.) transformant ainsi la conception du rapport traditionnel entre ceux-ci et leurs employeurs. Certains arguent qu'une telle logique est en-dehors du ressort des entreprises, compte tenu de leur mission prioritairement économique.
} 


\section{Le code « Responsabilité envers les employés » $(\mathrm{n}=203)$}

Le code « Responsabilité envers les employés » est associé à six autres codes (voir tableau 4 : Codes associés au code «Responsabilité envers les employés » $(\mathrm{n}=203)$ dont trois faisant partie de la famille «Emplois et employés» («Diversité main d'oeuvre/équité », «Formation et développement » et « Données sur les employés ») et y sont associés par un lien d'inclusion puisque les initiatives visant la diversité et l'équité et celle visant la formation et le développement professionnel, sont comprises dans les politiques et les pratiques que les banques estiment comme prioritaires dans la mise en cuvre de leur engagement en faveur de la responsabilité envers les employés; tandis que l'inclusion, dans les rapports, de données sur la performance, reflète l'importance que les banques accordent non seulement à leurs pratiques, mais aussi à l'évaluation des résultats de celles-ci. Les indicateurs de performance repérés dans les rapports des banques sont présentés au tableau 5 (voir Tableau 5 : Les indicateurs de performance utilisés par les banques canadiennes pour rendre compte de leurs résultats en matière de responsabilité envers les employés).

Tableau 4: Codes associés au code « Responsabilité envers les employés » $(\mathrm{n}=203)$

\begin{tabular}{|l|r|}
\hline Codes & Cooccurrences \\
\hline Diversité main d'oeuvre/équité & 64 \\
\hline Responsabilité envers la communauté & 63 \\
\hline Responsabilité envers les clients & 54 \\
\hline Formation et développement & 48 \\
\hline Vision RSE & 30 \\
\hline Données sur les employés & 24 \\
\hline
\end{tabular}

Deux autres codes («Responsabilité envers la communauté » et « Responsabilité envers les clients ») sont associés au code « Responsabilité envers les employés » par un lien de complémentarité, puisque ces trois types de responsabilité, ont été identifiés par les banques comme des dimensions incontournables de la RSE. D'autre part, on note aussi un lien de causalité entre la responsabilité envers les employés et celle envers les clients, puisque le discours des banques semble admettre que le développement des compétences des employés vise à s'assurer que leur façon d'offrir les services bancaires satisfasse les clients ${ }^{339}$. L'analyse de la

339 « À la BC2, le développement de notre force de gestion poursuit un double objectif. Il vise à mobiliser les employés performants en leur offrant une progression de carrière à la hauteur de leurs aspirations professionnelles et également à permettre à notre institution d'atteindre ses objectifs d'affaires, grâce à la présence d'un personnel mobilisé. Par le biais de l'Institut des leaders, en place 
cooccurrence des codes «Responsabilité envers les employés » et «Vision RSE » corrobore, quant à elle, la focalisation de la compréhension de la RSE sur les relations avec les principales parties prenantes, dont les employés, et sur la possibilité de conciliation de la recherche de rentabilité à long terme avec la $\operatorname{RSE}^{340}$.

Tableau 5: Les indicateurs de performance utilisés par les banques canadiennes pour rendre compte de leurs résultats en matière de responsabilité envers les employés

\begin{tabular}{|c|c|c|c|c|c|c|}
\hline & $\mathrm{BC} 1$ & $\mathrm{BC} 2$ & $\mathrm{BC} 3$ & $\mathrm{BC} 4$ & BC5 & BC6 \\
\hline Effectif & & & & & & \\
\hline Employés à temps plein/temps partiel & & & & & & \\
\hline Dépenses en salaires et avantages sociaux & & & & & & \\
\hline Dépenses en formation & & & & & & \\
\hline $\begin{array}{l}\text { Employés appartenant aux minorités } \\
\text { visibles }\end{array}$ & & & & & & \\
\hline Employés handicapés & & & & & & \\
\hline Femmes employées & & & & & & \\
\hline Taux de satisfaction des employés & & & & & & \\
\hline Accidents en milieu de travail & & & & & & \\
\hline Participants à l'actionnariat de la banque & & & & & & \\
\hline
\end{tabular}

Une cellule hachurée indique que l'indicateur a été utilisé par la banque.

depuis 1999, environ 450 gestionnaires seniors de la BC2 ont assisté à ce jour à des forums ayant pour objectif de clarifier la vision, la culture de gestion et les stratégies d'affaires de la Banque. (...). Devant un tel succès, l'Institut a décidé en 2001 de rendre ses programmes accessibles aux gestionnaires intermédiaires. (...). Aussi, pour la troisième année consécutive, plus de 250 de nos gestionnaires ont participé à la Journée du leadership, une autre initiative découlant de l'Institut des leaders, qui s'est déroulée en 2002 sous le thème «La satisfaction du client au centre de l'organisation ». Lors de cette journée de formation, les gestionnaires ont l'occasion, entre autres, de discuter avec les membres de la direction et d'entendre des conférenciers renommés qui traitent de sujets touchant des enjeux importants pour la Banque. Cette année, les conférences ont porté sur l'importance du rôle des gestionnaires dans l'implantation d'une culture de qualité de service ainsi que de leur impact sur la mobilisation des employés et la satisfaction des clients et des actionnaires. », (BC2).

340 « A la BC4, nous visons la viabilité à long terme de notre entreprise tout en contribuant au bien-être actuel et futur de notre nation. », (BC4).

« Offrir un service à la clientèle supérieur, consolider notre réputation en tant qu'employeur de premier ordre et contribuer à la santé globale et au bien-être de nos collectivités, voilà autant d'éléments importants par lesquels nous mesurons notre succès - sans oublier, bien entendu, créer une valeur sûre et à long terme pour nos actionnaires. », (BC5). 


\section{Le code « Diversité main d'œuvre/ équité » $(n=79)$}

Rappelons que les initiatives visant à tenir compte de la diversité des employés, des besoins spécifiques de certains groupes et l'équité envers tous les employés, sans égard aux groupes auxquels ils appartiennent, représentées par le code «Diversité main d'œuvre/ équité », constituent une des principales catégories de pratiques considérées comme responsables envers les employés par les banques. La relation d'inclusion de ce code avec le code "Responsabilité envers les employés », que nous avons identifiée précédemment se confirme. D'autre part, sa finalité se précise puisque le discours des banques reflète la focalisation sur un objectif stratégique, notamment celui de «bien servir les clients », donc de demeurer concurrentielles. Outre le code "Responsabilité envers les employés », quatre autres codes sont associés au code « Diversité main d'œuvre/ équité 》 («Responsabilité envers les clients », « Responsabilité envers la communauté », «Inclusion sociale/ diversité client », «Partenariat avec OBNL »). La présence des codes «Responsabilité envers les clients » et « Responsabilité envers la communauté » parmi ceux en cooccurrence fréquente avec le code «Diversité main d'œuvre/ équité » corrobore encore une fois la relation de causalité entre les responsabilités envers les clients et la communauté, d'une part, et celle avec les employés, d'autre part. Ainsi, reflétant la diversité de leurs clients ${ }^{341}$ et des membres de leur communauté, leur main d'œuvre diversifiée est considérée par les banques comme source d'avantage concurrentiel. Mais cette relation de causalité que nous venons de caractériser explique aussi le lien entre les codes «Diversité main d'œuvre/ équité » et «Inclusion sociale/ diversité client ${ }^{342}$. En énonçant leur objectif d'inclusion, les banques rappellent fréquemment la dimension stratégique qui veut qu'une telle initiative soit conciliable avec les objectifs commerciaux. Quant au code «Partenariat avec OBNL », dans les citations où il est en cooccurrence avec le code «Diversité main d'œuvre/ équité », il précise les moyens privilégiés par les banques dans leur démarche de promotion de la diversité et de l'équité, dont les partenariats avec des groupes communautaires ${ }^{343}$.

\footnotetext{
${ }^{341}$ « BC4 s'engage à assurer la diversité de sa main-d'œuvre parce que nous comprenons que pour réussir dans l'économie mondiale, nous devons concevoir des politiques et des initiatives qui favorisent la diversité, l'équité dans l'emploi et les différents talents et points de vue de nos employés », (BC4).

342 « BC1 offre un cadre de travail qui assure la compréhension et le respect de la diversité, ce qui est essentiel pour bien servir nos clients et pour attirer et conserver des employés. », (BC1).

343 « BC4 collabore avec des entreprises et des groupes collectifs autochtones afin de favoriser des relations constructives et permanentes et d'élaborer des approches afin de recruter des autochtones. $\bullet$ En collaboration avec la Federation of Saskatchewan Indian Nations et la Saskatchewan Indian Equity Foundation Inc., nous avons mis sur pied la Banque des Premières Nations du Canada en 1996. Aujourd'hui, environ $75 \%$ de son personnel est d'origine autochtone et, avec son expansion dans d'autres endroits, les occasions d'emploi augmenteront. - Afin de créer des occasions d'embauche pour les peuples autochtones, nous avons noué des liens avec de nombreux organismes, dont le Conseil canadien pour le commerce autochtone, l'Interprovincial Association for Native Employment et Miziwe Biik, un centre d'emploi pour autochtones de Toronto, afin de créer des occasions d'emploi pour
} 
Tableau 6: Codes associés au code « Diversité main d'œuvre/ équité » $(\mathrm{n}=79)$

\begin{tabular}{|l|r|}
\hline Codes & Cooccurrences \\
\hline Responsabilité envers les employés & 64 \\
\hline Responsabilité envers la communauté & 21 \\
\hline Partenariat avec OBNL & 21 \\
\hline Responsabilité envers les clients & 15 \\
\hline Inclusion sociale/diversité client & 11 \\
\hline
\end{tabular}

\section{Le code « Formation et développement » $(n=74)$}

Tableau 7: Codes associés au code « Formation et développement » $(\mathrm{n}=74)$

\begin{tabular}{|l|r|}
\hline Codes & Cooccurrences \\
\hline Responsabilité envers les employés & 48 \\
\hline Responsabilité envers les clients & 23 \\
\hline Rémunération et avantages & 11 \\
\hline
\end{tabular}

Trois codes sont associés au code «Formation et développement » (voir Tableau 7 : Codes associés au code "Formation et développement » $(\mathrm{n}=74))$. L'analyse de la cooccurrence du code «Formation et développement » avec les codes «Responsabilité envers les employés » et «Responsabilité envers les clients » ne révèle pas d'autres éléments de caractérisation supplémentaire mais confirme ses relations respectivement d'inclusion et de causalité avec les responsabilités envers les employés et les clients. Ainsi, c'est comme composantes d'un comportement responsable envers les employés visant à se doter d'une maind'œuvre qualifiée et à la hauteur des attentes des clients, que les initiatives telles l'offre d'outils d'apprentissage, la formation continue, les ententes de formation avec les institutions universitaires, ainsi que la divulgation d'information sur la performance à ces effets (par exemple, le montant annuel des dépenses en formation, le nombre d'employés diplômés dans le cadre des ententes avec les institutions universitaires) sont présentées dans les rapports des banques. La finalité stratégique de telles initiatives est ainsi corroborée ${ }^{344}$.

autochtones.», (BC4).

344 « Près de 800 employés de la $\mathrm{BC} 2$ ont décroché à ce jour un diplôme de premier ou de deuxième cycle grâce au Programme universitaire de BC2, fondé en 1996. Ce programme, unique dans l'industrie, est issu d'un partenariat avec l'Université du Québec à Montréal et les constituantes de l'Université du Québec, la Télé-université et l'Institut des banquiers canadiens. Il est taillé sur mesure pour répondre à nos besoins et à ceux de nos clients, ainsi qu'aux exigences des milieux financiers. (...). Cette formation universitaire reconnue permet aussi à nos employés d'augmenter leur valeur sur le marché de l'emploi. 
Au terme de cette analyse, il est possible d'esquisser un portrait de la structuration des codes de la famille «Emploi et employés », complétés par les autres codes associés à ceux les plus fréquents (Voir Schéma 1 : Structuration des codes de la famille «Emploi et employés »). Par le cadre «moyen/fin » dominant qui s'en dégage, ce portrait nous permet de répondre à la question de savoir si et dans quelles mesures les pratiques actuelles de RSE, plus précisément celles touchant l'emploi et les employés, ainsi que les moyens de les mesurer constituent un potentiel de changements dans les relations des banques avec leurs employés. Il apparaît, à la lumière de ce portrait, que la perspective de changements qui se dessine, du moins dans le discours des banques, est celui du passage d'une logique d'efficience minimaliste vers une logique de positionnement stratégique ${ }^{345}$. La logique plus progressiste d'imputabilité sociale semble encore absente du discours de l'ensemble des banques. Deux points principaux sont à relever à cet effet. D'une part, la focalisation du discours porte essentiellement sur les enjeux fortement réglementés, soit celui de l'inclusion à travers la promotion de la diversité et de l'équité, dans le sens de la lutte contre la discrimination et celui de la formation de la main-d'œuvre. Les préoccupations touchant l'emploi dans le Règlement sur la déclaration annuelle de 2002, viennent renforcer celles déjà exprimées par d'autres législations tant fédérales ${ }^{346}$ que provinciales ${ }^{347}$ au Canada. D'autre part, on note

(...). Le programme, qui représente à ce jour un investissement de plus de 10 millions de dollars, est périodiquement mis à jour afin de s'assurer que nos employés continuent de bénéficier d'une formation de qualité et toujours adaptée aux besoins changeants de notre environnement. », (BC2)

${ }^{345}$ Les résultats de cette recherche ne permettent pas de vérifier si au-delà du discours, les banques canadiennes ont vraiment adopté de nouvelles pratiques répondant à l'impératif de passage de la recherche de profit à court terme vers la construction d'avantages stratégiques, vecteurs de positionnement favorable, ou si les informations fournies dans leurs rapports ne sont qu'un recensement de données sur des activités qui existaient déjà et dont la divulgation est actuellement requise. Une telle évaluation requiert une observation plus poussée de ces pratiques, que la méthode d'analyse du contenu des rapports seule ne permet pas de réaliser.

${ }^{346}$ Outre le Règlement sur la déclaration annuelle de 2002, la Loi sur l'équité en matière d'emploi de 1995, qui vise à réaliser l'égalité en milieu de travail et à corriger les désavantages subis, dans le domaine de l'emploi, par les femmes, les autochtones, les personnes handicapées et les personnes qui font partie des minorités visibles, oblige chaque entreprise privée canadienne à soumettre annuellement un rapport indiquant les éléments suivants : a) les branches d'activité de ses salariés, le lieu de son établissement et le lieu de travail de ses salariés, le nombre de ceux-ci et celui des membres des groupes désignés qui en font partie; b) les catégories professionnelles qui composent son personnel et la représentation des membres de ces groupes dans chacune d'elles; c) les échelles de rémunération de ses salariés et la représentation des membres de ces groupes figurant à chacune d'elles ou à chacun de leurs échelons réglementaires; d) le nombre des recrutements, des avancements et des cessations de fonctions ainsi que, dans chaque cas, la représentation des membres des mêmes groupes.

${ }^{347}$ Un exemple de législation de niveau provincial qui oblige les entreprises à rendre compte de leurs activités en matière de formation de la main-d'œuvre est la Loi favorisant le développement de la formation de la main-d'œuvre du Québec, adoptée en 1995. Cette loi, communément appelée Loi du $1 \%$, oblige les entreprises ayant une masse salariale excédant un million de dollars à investir, au cours d'une même année civile, l'équivalent d'au moins $1 \%$ de cette masse salariale dans la formation du personnel et à déclarer le montant investi au ministère du Revenu du Québec. 
l'absence d'articulation entre les questions touchant l'emploi et les employés et la gestion des risques sociaux, soit la prise en compte des questions sociales dans les activités principales des banques, soit le financement et l'investissement. Alors que les banques sont des agents économiques dotés de ressources et de pouvoir substantiels, on s'attends à ce qu'elles soient des catalyseurs de changements en favorisant la responsabilisation des autres agents économiques, par leurs politiques d'achat et d'appels d'offre et par leurs politiques de prêt. Néanmoins, les banques canadiennes font peu état des exigences liées à l'emploi et aux employés qu'elles imposent à leurs entreprises fournisseurs, clientes et celles de leur portefeuille d'investissement. Notons que l'inclusion de critères sociaux, notamment ceux concernant le droit des travailleurs à l'étranger, dans les conditions de financement imposées par les banques, est encore du domaine auto-régulatoire.

Schéma 1 : Structuration des codes de la famille «Emploi et employés » ${ }^{348}$

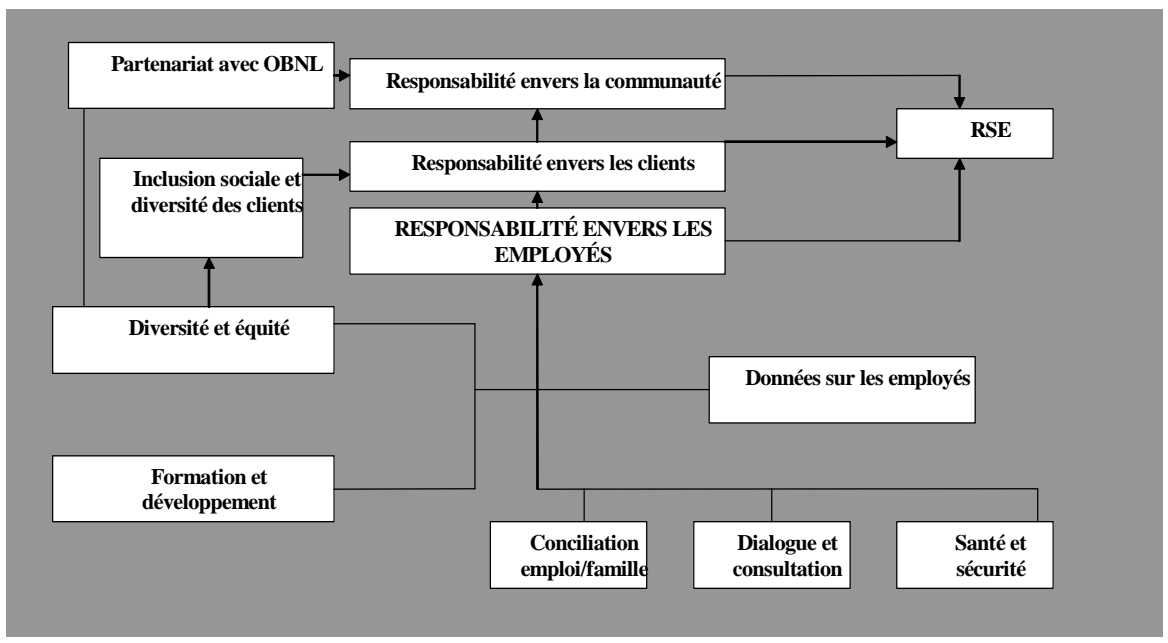

\section{CONCLUSION}

Rappelons le double objectif de ce travail, soit d'analyser dans quelles mesures l'évolution des connaissances sur la RSE (la théorie) offre une voie de renouvellement de la conceptualisation de la GSRH et, ensuite, d'examiner si les initiatives favorisées par les entreprises (la pratique) pour opérationnaliser ce qu'elles conçoivent comme leur responsabilité envers les employés peuvent être source de transformations transcendant la finalité strictement utilitaire.

\footnotetext{
${ }^{348}$ Les codes indiqués par les têtes de flèches sont indicateurs de la fin dans la relation moyen/fin.
} 
L'évaluation de la théorie nous a permis de conclure que le potentiel de renouvellement des connaissances sur la GSRH, à la lumière de celles sur la RSE, se trouve dans les approches appartenants aux courants du questionnement et de la compréhension empirique, qui ont été à la source d'études empiriques d'origines française, québécoise et scandinave au cours des cinq dernières années. Ces approches se distancent des approches prescriptives traditionnelles et proposent un nouvel éclairage basé sur des théories institutionnelles sociologiques et économiques favorisant des concepts tels les représentations sociales, la régulation, la construction sociale, l'institutionnalisation, etc.

L'évaluation de la pratique, à l'aide de l'étude du discours des banques canadiennes, a indiqué quant à elle que la perspective actuelle de changements qui se dessine est celui du passage d'une logique d'efficience minimaliste vers une logique de positionnement stratégique. Tout en présentant la gestion responsable des ressources humaines dans leurs rapports RSE comme un moyen de se constituer un avantage concurrentiel, les positionnant comme employeurs de choix et leur permettant de répondre aux attentes des clients, les banques canadiennes fournissent essentiellement des renseignements sur l'équité, la diversité et la formation de la main-d'œuvre dont la production est exigée par des législations auxquelles elles sont soumises. Cette tendance pourrait être vérifiée en analysant les rapports subséquents produits par ces mêmes banques. Il serait pertinent de comparer les résultats relativement aux banques canadiennes avec ceux provenant des bilans sociaux de banques d'autres pays.

On peut alors conclure de cette analyse, que tant en théorie qu'en pratique, la GSRH demeure encore dans une conceptualisation traditionnelle des ressources humaines, soit celle véhiculée par les tenants de la théorie des dépendances des ressources. Le temps est venu d'étudier les conditions institutionnelles, notamment les mécanismes régulateurs, qui pourraient être vecteurs de discours et de pratiques favorisant le développement social des travailleurs et des relations de travail répondant aux véritables impératifs sociaux. 


\section{BIBLIOGRAPHIE}

Analoui, Farhad (1998). Human Resource Management Issues in Developing Countries. Aldershot: Ashgate.

Andrews, M.C et K.K. Kacmar (2001). « Discriminating amongst organizational politics, justice and support ». Journal of Organizational Behavior, vol. 18 no 6, p. 347-366.

Armstrong, M. et A. Baron (2000). Performance Management - The New Realities. CIPD: Londres.

Aupperle, K.E., A.B. Carroll et J.D. Hatfield (1985). «An empirical investigation of the relationship between corporate social responsibility and profitability ». Academy of Management. The Academy of Management Review, vol. 28, p. 446-463.

Bardelli, Pierre. 2006. « La Responsabilité Sociale des Entreprises, argument de régulation post-fordienne et/ou support de micro-régularités ». Les cahiers de la de Chaire de responsabilité sociale et de développement durable, no 01-2006, 32 p.

Barney, J.B. (1991). « Firm Resources and Sustained Competitive Advantage ».

Journal of Management, vol. 17, no. 1, p. 99-120.

Barrett, E. (1999). « Justice in the workplace? Normative ethics and the critique of human resource management ». Personnel Review, vol. 28 no 4, p. 307318.

Becker, Brian, Mark A. Huselid, P.S. Pickus et M.F. Spratt (1997). « Human resource as a source of shareholder value: Research and recommendations ». Human Resource Management, vol. 36 no 1, p. 39-47.

Beer, M. (1997). « The transformation of the human resource function: Resolving the tension between a traditional administrative and a new strategic role ». Human Resource Management, vol. 36 no 1 , p. 49-56.

Bontis, N. (1996). « There's a price on your head: Managing intellectual capital strategically ». Business Quarterly, vol. 60 no 4, p. 40-47.

Bowen, Howard (1953). Social Responsibilities of the Businessman. New York: Harper and Row.
Boxall Peter. (1996). " The strategic HRM debate and the resource-based view of the firm ». Human Resource Management Journal, vol. 6 no 3, p. 5975.

Boxenbaum, Eva (2006). « Corporate social responsibility as institutional hybrids ». Journal of Business Strategies, vol. 23 no 1, p. 45-63.

Carroll, Archie B. (1979). « A threedimensional conceptual model of corporate performance ». Academy of Management. The Academy of Management Review, vol. 4 no 4, p. 497-506.

Carroll, Archie B. (2000). «A Commentary and Overview on Key Questions on Corporate Social Performance Measurement ». Business and Society, vol. 39 , no 4, p. 466-478.

Champion, Emmanuelle, Corinne Gendron et Alain Lapointe (2005). « Les représentations de la responsabilité sociale des entreprises : un éclairage sociologique ». Les cahiers de la Chaire de responsabilité sociale et de développement durable, no 05-2005, 24 $\mathrm{p}$.

Clarkson, Max (1995). «A Stakeholder Framework for Analyzing and Evaluating Corporate Social Performance ». Academy of Management. The Academy of Management Review, vol. 20 no 1, p. 92-117.

Colbert, Barry A. et Elizabeth C. Kuruez (2007). « Three conceptions of triple bottom line business sustainability and the role for HRM ». HR. Human Resource Planning, vol. 30 no 1, p. 2129.

Cramer, Jacqueline, Jan Jonker et Angela van deir Heijden (2004). " Making sense of corporate social responsibility ». Journal of Business Ethics, vol. 55 no 2, p. 215-222.

Daft, R.L. et K.E. Weick, K.E. (1984). «Toward a model of organizations as interpretation systems ». Academy of Management. Academy of Management. The Academy of Management Review, vol. 9 no. 2, p. 284-95.

Deetz, Stanley (1996) « Describing 
Differences in Approaches to Organizational Science: Rethinking Burrell and Morgan and their Legacy », Organization Science, vol. 7, p. 191-207

De Serres Andrée, Corinne Gendron et Lovasoa Ramboarisata (2006). « Pratiques et stratégies des institutions financières en matière de divulgation d'information sur leur responsabilité sociale ». Cahier conjoint de la de Chaire de responsabilité sociale et de développement durable et du Groupe interdisciplinaire de recherche en éthique financière, no 16-2006, $170 \mathrm{p}$.

De Serres, Andrée (2005). «Une analyse comparative des stratégies d'intervention des législateurs en matière de gouvernance éthique et de responsabilité sociale des entreprises aux États-Unis, au Royaume-Uni et en France », Revue des Sciences de la Gestion, avril.

Delery, John E. et Harold D. Doty (1996). « Modes of theorizing in strategic human resource management: tests of universalistic, contingency, and configurational performance predictions ». Academy of Management Journal, vol. 39 no 4, p. 802-835.

Donaldson, T. et L. Preston (1995). "The stakeholder theory of the corporation: concepts, evidence, and implications ». Academy of Management. The Academy of Management Review, vol. 20, p. 6391.

Edvinsson, L. et M.S. Malone (1997). Intellectual Capital: Realizing Your Company's True Value by Finding its Hidden Brainpower. New York: Harper Business.

Elkington, John (1994). « Towards the sustainable corporation: Win-win business strategies for sustainable development ». California Management Review, vol. 36 no 2, p. 90-100.

Elkington, John (1997). Cannibals With Forks: The Triple Bottom Line of $21^{\text {st }}$ Century Business. Oxford: Capstone Publishing.

Fombrun, C.J., N.M. Tichy et M.A. Devanna (1984). Strategic Human Resource Management. New-York: Wiley \&
Sons.

Freeman, Edward (1984). Strategic Management: a Stakeholder Approach. Boston: Pitman.

Gendron, Corinne (2000). « Le questionnement éthique et social de l'entreprise dans la littérature managériale ». Cahiers du CRISES, sous la dir. de P. Bélanger et B. Lévesque, Collection Études théoriques, no ET0004.

Gendron, Corinne (2002). « Envisager la responsabilité sociale dans le cadre des régulations portées par les nouveaux mouvements sociaux économiques ». Les cahiers de la Chaire de responsabilité sociale et de développement durable, no 01-2002, 29 $\mathrm{p}$.

Gendron, Corinne (2005). « Les codes d'éthique: de la déontologie à la responsabilité sociale ». Les cahiers de la Chaire de responsabilité sociale et de développement durable, no 03-2005, 33 $\mathrm{p}$.

Gendron, Corinne, Alain Lapointe et MarieFrance Turcotte (2003). " Codes de conduite et entreprise mondialisée : Quelle responsabilité sociale? Quelle régulation?». Les cahiers de la Chaire de responsabilité sociale et de développement durable, no 12-2003, 33 $\mathrm{p}$.

Gendron, Corinne, Alain Lapointe et MarieFrance Turcotte (2004). « Responsabilité sociale et régulation de l'entreprise mondialisée». Relations industrielles, vol. 59 no 1, p73-98.

Gond, Jean Pascal et Olivier Herrbach (2006). «Social Reporting as an Organisational Learning Tool? A Theoretical Framework ». Journal of Business Ethics, vol. 65 no 4, p. 359-371.

Greenwood, Michelle R. (2002). « Ethics and HRM: A review and conceptual analysis ». Journal of Business Ethics, vol. 36, p. 261-278.

Griffin, Jennifer J. et John F. Mahon (1997). « The corporate social performance and corporate financial performance debate: Twenty-five years of incomparable research ». Business \& Society, vol. 36 no 1, p. 5-31. 
Guest, David. (1987). « Human resource management and industrial relations », Journal of Management Studies, vol. 24 no 5, p. 503-521.

Guest, David. (1997). « Human resource management and performance: A review and research agenda », International Journal of Human Resource Management, vol. 8 no 3, p. 263-276.

Guest, David. (1999). « Human Resource Management the Workers' Verdict », Human Resource Management Journal, vol. 9 no 3, p. 5-25.

Hart, T.J. (1993). « Human resource management: Time to exercise the militant tendency 》. Employee Relations, vol. 15 no 3, p. 29-36.

Hill, J. (2001). « Thinking about a more sustainable business - An indicators approach ». Corporate Environmental Strategy, vol. 8 no 1 , p. 30-38.

Huber, G.P. (1991), « Organisational learning: the contributing process and the literatures ». Organisation Science, vol. 2 no. 1 , p. $88-115$.

Jonker, Jan, Jacqueline Cramer et Angela van deir Heijden (2004). « Developing meaning in action: (Re)constructing the process of embedding corporate social responsibility (CSR) in companies ». ICCSR (International Centre for Corporate Social Responsibility) Research Paper Series, no 16-2004, 19 $\mathrm{p}$.

Karami, Azhdar, Farhad Analoui et John Cusworth (2004). « Strategic human resource management and resourcebased approach: The evidence from the British manufacturing industry ». Management Research News, vol. 27 no 6, p. 50-68.

Keenoy, Tom (1990a). « HRM: Rhetoric, Reality and Contradiction ", The International Journal of Human Resource Management, vol. 1 no 3, p. 363-384.

Keenoy, Tom (1990b). « Human resource management: a case of the wolf in sheep's clothing », Personnel Review, vol. 19 no 2, p. 3-9.

Lahteenmaki, S. J. Storey et S. Vanhala (1998). « HRM and company performance: The use of measurement and the influence of economic cycles ». Human Resource Management Journal, vol. 8 no 2, p. 51-65.

Lansisalmi, H., J-M Peiro et Mika Kivimaki. 2004. " Grounded theory in organizational research ». In Essential Guide to Qualitative Methods in Organizational Research, sous la dir. de Catherine Cassell et Gillian Symon, p.242-255. Thousand Oaks: Sage Publications.

Legge, Karen (1996). « Morality bound ». People Management Journal, vol. 25 no 2, p. 34-36.

Lemons, M.A et C.A. Jones (2000). « Procedural justice in promotion decisions: Using perceptions of fairness to build employee commitment ». Journal of Managerial Psychology, vol. 16 no 4, p. 268-280.

Litz, Reginald (1996). «A resource-based view of the socially responsible firm: stakeholder interdependence, ethical awareness, and issue responsiveness as strategic assets ». Journal of Business Ethics, vol 15 no 12, p. 1355-1363.

Logsdon, Jeanne et Donna Wood (2002). « Business citizenship: From domestic to global level of analysis ». Business Ethics Quarterly, vol. 12 no 2, p. 155188.

Lundy, O. (1994). « From personnel management to strategic human resource management ». The International Journal of Human Resource Management, vol. 5 no 3, p. 687-717.

Mabey. C., G. Salaman et J. Storey (1998). Strategic Human Resource Management. Londres: SAGE Publications.

McWilliams, Abagail, Donald Siegel et Patrick Wright (2006). " Corporate Social Responsibility: Strategic Implications ». Journal of Management Studies, vol 43 no 1, p. 1-18.

Miller, P. (1989). « Strategic human resource management: What it is and what it isn't? ». Personnel Management, février, p 46-51.

Miller, Paul (1996). « Strategy and the ethical management of human resources ». Human Resource Management Journal, vol. 6 no 1, p. 5-18. 
Mitchell, Ron, Bradley Agle et Donna Wood (1997). « Toward a theory of stakeholder identification and salience: Defining the principle of who and what really counts ». Academy of Management. The Academy of Management Review, vol. 22 no 4, p. 853-886.

Nahapiet, J. et S. Ghoshal (1998). « Social capital, intellectual capital, and the organizational advantage ». Academy of Management. The Academy of Management Review, vol. 23, p. 242266.

Newell, A. et H.A. Simon (1972). Human Problem Solving. Englewood Cliffs: Prentice-Hall.

Oliver, A.G. (2001). " Can sustainable development and the market co-exist? ». Corporate Environmental Strategy, vol. 8 no 1 , p. 24-29.

Penrose, Edith (1959). The Theory of the Growth of the Firm. Oxford: Oxford University Press.

Peters, T. et R. Waterman (1982). In Search of Excellence. New York: Harper \& Row.

Pettijohn, L.S, S. Parker, C.E. Pettijohn et J.L. Kent (2001). «Performance appraisal: Usage, criteria and observations ». Journal of Management development, vol. 20 no 9, p. 754-771.

Post, James., Lee Preston et Sybille Sachs (2002). « Managing the extended enterprise: The new stakeholder view ». California Management Review, vol. 45 no 1, p. 6-28.

Preston, Lee (1998). « Agents, stewards, and stakeholders ». Academy of Management. The Academy of Management Review, vol. 23 no 1, p. 9.

Preston, Lee et James Post (1974). « The third managerial revolution ». Academy of Management Journal, vol. 17 no 3, p. 476-486.

Preston, Lee et Thomas Donaldson (1999). « Stakeholder management and organizational wealth ». Academy of Management. The Academy of Management Review, vol. 24 no 4, p. 619-620.

Reed, Darryl (1999). « Stakeholder management theory: a critical theory perspective ». Business Ethics
Quarterly, vol. 9 no 3, p. 453-483.

Russo, Michael et Paul Fouts (1997). « A resource-base perspective on corporate environmental performance and responsibility ». Academy of Management Journal, vol 40 no 3, p. 534-559.

Sahlin-Andersson, Kerstin (2006). « Corporate social responsibility: A trend and a movement, but of what and for what? ». Corporate Governance, vol. 6 no 5, p. 595-608.

Sennett, R. (1999). « How Work Destroys Social Inclusion ». New Statesman, vol. 128, p. 25-27.

Sethi, Prakash (1975). « Dimensions of corporate social performance: An analytic framework ». California Management Review, vol. 17, p.58-64.

Shrivastava, Paul (1987). « Rigor and practical usefulness of research in strategic management ». Strategic Management Journal, vol. 8 no 1, p. 77 92.

Simmons, John (2003). « Balancing performance, accountability and equity in stakeholder relationships: Towards more socially responsible HR practice ». Corporate-Social Responsibility and Environmental Management, vol. 10 no 3, p. 129-140.

Steurer, Reinhard, Markus Langer, Astrid Konrad et Andre Martimuzzi (2005). « Corporations, stakeholders and sustainable development I: A theoretical exploration of business-society relations ». Journal of Business Ethics, vol. 61, p. 263-281.

Stewart, T.A (1997). Intellectual Capital. New York: Doubleday.

Thomas, Kenneth et Walter Tymon Jr (1982). « Necessary properties of relevant research: Lessons from recent criticisms of the organizational sciences ». Academy of Management. The Academy of Management Review, vol. 7 no 3, p. 345-357.

Townley, B. (1993). « Foucault, Power/Knowledge, and Its Relevance for Human Resource Management ». Academy of Management. The Academy of Management Review, vol.18 no 3, p. 518-546. 
Vuontisjarvi, Taru (2006). « Corporate social reporting in the European context and human resource disclosures: An analysis of Finnish companies ». Journal of Business Ethics, vol. 69, p. 331-354.

Wartick, Steven et Philip Cochran (1985). « The evolution of the corporate social performance model ». Academy of Management. The Academy of Management Review, vol. 10 no 4, p. 758-769.

Weick, Karl E. 1995. Sense-making in Organizations. Sage Publications.

Winstanley, D. J. Woodall et E. Heery (1996). «Business ethics and human resource management ». Personnel Review, vol. 25 no 6, p. 5-12.

Wirtenberg, Jeana, Joel Harmon, William G. Russell et Kent D. Fairfield (2007). « HR's role in building a sustainable enterprise: Insights from some of the world's best companies ». HR. Human Resource Planning, vol. 30 no 1, p. 1020.

Wood, Donna (1991). « Corporate social performance revisited ». Academy of Management. The Academy of Management Review, vol. 16 no 4, p. 691-718.

Wood, Donna (2004). « On global business citizenship: Introduction to the special issue ». Business and Society Review, vol. 109 no 1, p. 1-3.

Wood, Donna et Jeanne Logsdon (2002). « Business citizenship: From individuals to organizations ». In Ruffin Series in Business Ethics, p. 59-94.

Youndt, M.A, S.A. Snell, J.W. Dean et D.P. Lepak (1996). « Human resource management, manufacturing strategy, and firm performance ». Academy of Management Journal, vol. 39, p. 836866.

Youndt, Mark A. et Scott A. Snell (2004). « Human resource configuration: Intellectual capital, and organizational performance ». Journal of Managerial Issues, vol. 16 no 3, p. 337-360 


\section{Ouvrages des auteurs}

- De Serres, A., \& Roux, M., L'impact des stratégies de responsabilité sociale des banques corporatives sur la mission et la gouvernance des institutions financières coopératives dans La gouvernance des entreprises coopératives. Sous la direction de Murnoz, J. ; Radrigan, M., \& Regrand, Y., Presses Universitaires de Rennes

- De Serres, A., 2007, Un défi pour l'ingénierie financière de projet : savoir transférer et gérer les risques des concessions d'infrastructure publique. L'Harmattan, Paris, France.

- De Serres, A., .2005, La responsabilité sociale des banques : utopie ou réalité ? Exclusion et liens financiers. Rapport Centre Walras 2004. Université Lumière Lyon 2. Economica.

- De Serres, A., 2001, Annuaire du Québec 2001, L'impact de la mondialisation sur les services financiers au Québec, Fides.

- De Serres, Andrée, Annuaire du Québec, 2000, Faits saillants dans l'industrie des services financiers au Québec de juillet 1999 au 30 juin 2000. 